\title{
GENERAL THEORY OF MODULAR INVARIANTS*
}

\author{
BY
}

\section{LEONARD EUGENE DICKSON}

\section{Introduction.}

The discovery of the fundamental theorems, established in the first part of the present paper, on the invariants of a general system of $s$ forms under linear transformations in a finite field was the outcome of a new standpoint for the consideration of modular invariants. In former papers on the subject (cited later), the test for the invariance of a polynomial consisted in a more or less direct verification that it remained unaltered, up to a power of the determinant of the transformation, under the general linear group $G$ in the field; instead of certain generators of the latter, the corresponding annihilators were employed. In the present paper, the transformation concept is employed only to furnish a complete set of non-equivalent classes $C_{0}, \ldots, C_{f-1}$ of systems of $s$ forms under the group $G$. Thus the test for the absolute invariance of a polynomial $P$ is that $P$ shall take the same value for all systems of 8 forms in a class. It is shown in $\S 4$ that the number of linearly independent absolute invariants equals the number $f$ of classes under the total group $G$. In $\$ 6$ it is shown that the number of linearly independent invariants, including both absolute and relative, equals the number of classes under the group $G_{1}$ of transformations of determinant unity; it is furthermore specified which of the invariants under $G_{1}$ are invariants of the $s$ forms.

The general theory is applied in $\S \S 8,9,16-19$ to the determination of all the invariants of the general $m$-ary quadratic form in the Galois field of order $p^{n}$ and in $\$ \$ 22-26$ to the construction of all invariants of the binary cubic form $\uparrow$ in the $G F\left[p^{n}\right]$. For the practical construction of the invariants, there is developed a uniform process, of function-theoretic nature, for the conversion of non-invariantive characterizations of the classes into invariantive characterizations. The intervening sections are devoted to the determination and characterization of the classes of the forms under investigation. A mere list of canonical types of forms is not sufficient. For $m$-ary quadratic forms in the $G F\left[2^{n}\right]$ such a list has been given $\ddagger$ by the author; to obtain necessary and

* Presented to the Society (Chicago), January 1, 1909.

$\dagger$ Results for higher binary forms and for a pair of $m$-ary quadratio forms are to be published soon.

† American Journal of Mathematios, vol. 21 (1899), p. 222. 
sufficient criteria for each class, a new theory for such forms has been constructed in $\S \S 10-15$. Also for binary cubic forms, the case ( $\$ 26)$ in which the modulus $p$ equals 2 is more intricate than the general case $p>2$. The nature of the invariants is quite different in the two cases, a result to be anticipated for quadratic forms, but rather surprising for cubic forms. The consequent assignment of such a large part of the present paper to the special case $p=2$ was made not merely for the sake of completeness, but rather on account of the very prominent rôle which the linear groups with modulus 2 play in the applications * to geometry and in the general theory of linear groups.

Invariantive properties are often expressed in the algebraic theory by the vanishing of a covariant, or by means of an integer such as the rank of the matrix of a quadratic form. In the modular theory, every such property can be expressed by means of an explicit invariantive function of the coefficients. For quadratic forms illustrations of this point occur below. For a covariant $K$ with the coefficients $A_{i}$,

$$
I=\Pi\left(1-A_{i}^{p n-1}\right)
$$

is a modular invariant of the initial forms, in view of formula (1). We have $K \equiv 0$ or $K$ 三 0 according as $I=1$ or $I=0$.

The method of the present paper for constructing modular invariants affords immediately important interpretations of these invariants.

It seems probable that modular invariants are destined to play a rôle in the theory of numbers comparable to that played by algebraic and differential invariants in higher algebra and geometry.

For a given set of forms, the theory of its modular invariants presents a doubly infinite number of problems, in view of the order $p^{n}$ of the finite field. As compared with the use of annihilators employed in the earlier papers, the power of the present method may be inferred from the ease with which the various fields are now considered simultaneously. Hitherto the completeness of a proposed system of modular invariants for an infinitude of fields had not been established, even in so apparently simple a case as that of the binary quadratic form.

\section{Existence theorems on modular invariants, §§ 1-5.}

1. We shall make use of the following general theorem on interpolation in any finite field. Within the $G F\left[p^{n}\right]$, there exists one and but one polynomial $\phi\left(x_{1}, \ldots, x_{k}\right)$ which has each exponent $\leqq p^{n}-1$ and which takes prescribed values $v_{x_{1}, \ldots, x_{k}}$ for every set of elements $x_{1}, \ldots, x_{k}$ in the field.

To proceedfby induction, let the theorem be true for $k-1$ variables $x_{2}, \ldots, x_{k}$. Denote the elements of the field by $e_{0}, e_{1}, \ldots, e_{\nu}$, where $\nu=p^{n}-1$. Then, for each value of $i$, there exists an unique polynomial $\phi\left(e_{i}, x_{2}, \cdots, x_{k}\right)$ with expo-

* JoRdar, Traité des substitutions, p. 313, p. 329 ; Dickson, 1 nnals of Mathematios, ser. II, vol. 6:(1905),:pp. 141-150. 
nents $\leqq \nu$, which takes the values $v_{\epsilon_{1}, x_{3}, \ldots, x_{k}}$ when $x_{2}, \ldots, x_{k}$ range over the field. W.e may now determine uniquely polynomials $\psi_{i}$ in $x_{2}, \ldots, x_{k}$ such that

$$
\psi_{0}+\psi_{1} x_{1}+\cdots+\psi_{\nu} x_{1}^{\nu} \equiv \phi\left(x_{1}, x_{2}, \cdots, x_{k}\right)
$$

will take the prescribed values $v_{x_{1}, \ldots, x_{k}}$. Indeed, the equations obtained by setting $x_{1}=e_{0}, \cdots, e_{v}$ uniquely determine $\psi_{0}, \cdots, \psi_{\nu}$ as linear functions of the known polynomials $\phi\left(e_{i}, x_{2}, \cdots, x_{k}\right)$, since

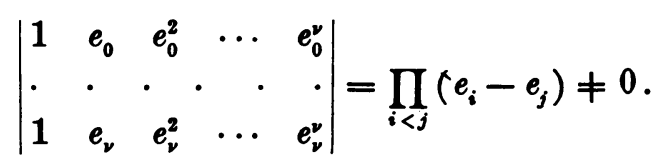

The argument applies also when $k=1$, the $\psi_{i}$ being parameters in the field. Hence the induction is complete.

Corollary. If two polynomials, with each exponent $\leqq p^{n}-1$, are equal in $G F\left[p^{n}\right]$ for all sets of values of the variables, they are identical.

2. Consider a system of forms $F_{1}, \ldots, F_{\text {, }}$, where $F_{i}$ is the general polynomial of degree $d_{i}$ in $m$ variables having as coefficients arbitrary parameters in the $G F\left[p^{n}\right]$. Assigning particular values to these parameters, we obtain a particular system $S_{1}$ of $s$ forms. The distinct systems that can be obtained from $S_{1}$ by applying the various transformations of a given $m$-ary linear homogeneous group* $L$ in the $G F\left[p^{n}\right]$ constitute the class $C_{1}$ of systems of forms conjugate, under $L$, with the given system $S_{1}$. Since the field is of finite order $p^{n}$, the order of $L$ is finite, the number of systems in one class is finite, and there is a finite number of classes $C_{0}, C_{1}, \ldots, C_{f-1}$.

Selecting arbitrarily a system $S_{i}$ from the class $C_{i}$, we shall say that $S_{0}, \ldots$, $S_{f-1}$ constitute a complete set of non-equivalent systems, under $L$, of $s$ forms of degrees $d_{1}, \ldots, d_{i}$. When the $S_{i}$ are relatively simple representatives of their classes, they are said to form a complete set of canonical types under $L$.

We shall expressly include the class, henceforth designated by $C_{0}$, which is composed of the system of forms all of whose coefficients are zero (see end of $§ 4$ ).

3. ThEOREM. Under a given linear homogeneous group $L$ in the $G F\left[p^{n}\right]$, $a$ system of forms has one and but one invariant $\dagger$ which takes prescribed values $v_{0}, \cdots, v_{f-1}$ for the various classes $C_{0}, \ldots, C_{f-1}$.

By $\S 1$, there exists one and but one polynomial $P$ which takes the prescribed values. If any chosen transformation of $L$ replaces $P$ by $P^{\prime}$, then $P^{\prime}$ takes the same values, so that $P^{\prime} \equiv P$.

* The replacement of a group by any set of transformations is only an apparent generalization.

$\dagger \Delta$ polynomial in the coefficients $a_{j}$ of the forms, each $a$ entering to a power $\leqq p^{n}-1$, as may be assumed in view of $a^{p^{n}}=a$. 
4. Denote by $I_{k}$ the uniquely determined invariant which has the value unity for the class $C_{k}$ and the value zero for every class $C_{i}(i \neq k)$. Since $I_{k}$ completely characterizes the class $C_{k}$, it will be called the characteristic invariant of the system of forms for the class $C_{k}$ under the group $L$.

Let $\sum c_{i} I_{i} \equiv 0$ be a linear homogeneous relation between the invariants $I_{i}$, with constant coefficients $c_{i}$ in the $G F\left[p^{n}\right]$. Assign to the coefficients $a_{j}$ of the given system of forms the values which they have in a particular system of forms belonging to the class $C_{k}$. Then $I_{k}=1, I_{i}=0(i \neq k)$. Hence $c_{k}=0$. Thus the characteristic invariants are linearly independent in the field.

Consider any invariant $I$ of the system of forms under the group $L$. Let $I$ have the value $v_{i}$ for the class $C_{i}$. Then, by the corollary in $\S 1, I \equiv \sum v_{i} I_{i}$. Any invariant is a linear homogeneous function of the characteristic invariants with constant coefficients in the field.

The essential part of the preceding results is contained in the

TheOREM. The number of linearly independent invariants of a given system of 8 forms under a given linear homogeneous group $L$ in the $G F\left[p^{n}\right]$ equals the number of non-equivalent classes under $L$.

We note the non-homogeneous relation $\sum I_{i}=1$. To compensate for the inclusion of the trivial invariant unity in counting the number of linearly independent invariants, we note that we have also included the trivial class $C_{0}$ of identically vanishing forms. For the latter,

$$
I_{0}=\prod_{i=1}^{\sigma}\left(1-a_{i}^{p n-1}\right),
$$

where $a_{1}, \cdots, a_{\sigma}$ is the aggregate of the coefficients of the $s$ forms.

5. Theorem. Any set of $f-1$ of the $f$ characteristic invariants are independent, in the sense that no invariant of such a set equals a rational integral function of the remaining invariants of that set.

Let the given set contain the $I_{j}(j \neq g)$, and assume that $I_{k}$ equals a polynomial $P$ in the $I_{j}(j \neq g, k)$. Since $I_{i}^{2}=I_{i}, I_{i} I_{j}=0(i \neq j), I_{k}=P$ may be given the form $I_{k}=c+\sum c_{j} I_{j}(j \neq g, k)$. Replace $c$ by $c \sum I_{i}$. The resulting homogeneous relation must be an identity. But, by the coefficients of $I_{k}$ and $I_{g}, c=1, c=0$, respectively.

To give a second proof, consider representatives $S_{k}$ and $S_{g}$ of the classes $C_{k}$ and $C_{g}(k \neq g)$. For the particular values of the coefficients in $S_{k}$ and those in $S_{g}$, the invariant $I_{k}$ takes different values ( 1 and 0 ), whereas each invariant $I_{i}(i \neq k, i \neq g)$ takes the same value zero. Hence $I_{k}$ cannot equal a polynomial in the $I_{i}(i \neq k, g)$. 
Absolute and relative invariants of a system of forms.

6. When $L$ is the group $G$ of all $m$-ary linear homogeneous transformations in the $G F\left[p^{n}\right]$, the invariants defined in $\$ \S 3,4$ are called the absolute invariants of the 8 forms. When $L$ is the group $G_{1}$ of all transformations of determinant unity, those invariants of $G_{1}$ which are multiplied by $\Delta^{*}$ under every transformation of determinant $\Delta$ are called the relative invariants of weight 20 of the $s$ forms. A knowledge of the classes of forms under $G_{1}$ will be shown to be sufficient for the construction of all relative invariants.

Each class $C_{i}$ under $G$ separates into certain classes $C_{i 1}, C_{i 2}, \ldots, C_{i q_{i}}$ under $G_{1}$, which are transformed amongst themselves by all transformations of the $p^{n}-1$ possible determinants. Hence $e_{i}=\left(p^{n}-1\right) / g_{i}$ is the number of distinct determinants of the automorphs of a class $C_{i}$. These $e_{i}$ distinct values may be expressed as powers $\rho^{\mu}, \rho^{\mu^{\prime}}, \ldots$ of a primitive root $\rho$ of the field, where each exponent is $\geqq 0$. Let $\mu$ be the minimum positive exponent. Then $\mu^{\prime}=k \mu+\nu(0 \leqq \nu<\mu)$. Let $T$ and $M$ be automorphs of determinants $\rho^{\mu^{\prime}}$ and $\rho^{\mu}$, respectively. The determinant of $T M^{-k}$ is $\rho^{\nu}$. Hence $\nu=0$, so that every exponent is a multiple of $\mu$. There are $\left(p^{n}-1\right) / \mu$ distinct powers of $\rho^{\mu}$. Hence $\mu=g_{i}$ and the $e_{i}$ distinct determinants of the automorphs of $C_{i j}$ are the distinct powers of a primitive root $\rho^{g_{i}}$ of $x^{e_{i}}=1$.

Under a linear transformation $R$ of determinant $\rho$, the classes $C_{i 1}, \ldots, C_{i g_{i}}$ undergo a permutation $P$. Since the determinant of $R^{\natural}$ is a root of $x^{4}=1$, $P_{01}$ is the identity. Since $\rho^{a t}$ is the least positive power of $\rho$ which gives a root of $x^{\varepsilon_{i}}=1, P^{0_{i}}$ is the least positive power of $P$ which leaves a class $C_{i v}$ unaltered. Hence $\boldsymbol{P}$ permutes $C_{i 1}, \ldots, C_{i q_{i}}$ in a single cycle. By assigning a suitable sequence to the $C_{i j}\left(j=1, \cdots, g_{i}\right)$, we may set

$$
P=\prod_{i}\left(C_{i 1} C_{i 2} \ldots C_{i i_{i}}\right) \text {. }
$$

Let $a_{t}$ be the general coefficient of the given system of forms, $a_{t}^{\prime}$ the corresponding coefficient of the forms obtained by applying the transformation $R$. Thus $R$ transforms a function $V(a)$ of the coefficients $a_{t}$ into $V\left(a^{\prime}\right)=V^{\prime}(a)$. Let $V$ be an invariant under the group $G_{1}$, so that $V(a)$ has the same value for all systems of forms in a class $C_{i j}$. Now $R$ transforms the class $C_{i j}$ into the class $C_{i+1}$, which is to be identified with $C_{i 1}$ when $j=g_{i}$. Hence the value of $V$ for the class $C_{i j+1}$ equals the value of the transformed function $V^{\prime}$ for the class $C_{i j}$. Let $V$ be the characteristic invariant $I_{i j+1}$, so that $I_{i j+1}$ has the value unity for the class $C_{i j+1}$ and the value zero for all the remaining classes under $G_{1}$. Hence $I_{i j+1}^{\prime}$ equals unity for $C_{i j}$ and zero for the remaining classes. Thus $I_{i j+1}^{\prime}$ equals the characteristic invariant $I_{i j}$ for the class $C_{i v}$. Hence

$$
R \sim \prod_{i}\left(I_{i g_{i}} \ldots I_{i j+1} I_{i j} \ldots I_{i 2} I_{i 1}\right)
$$


Now $\gamma_{i}=\rho^{e_{i}}$ belongs to the exponent $g_{i}$. The $g_{i}$ functions

$$
\Sigma_{i k}=I_{i 1}+\gamma_{i}^{k} I_{i 2}+\gamma_{i}^{2 k} I_{i 3}+\cdots+\gamma_{i}^{\left(g_{i}-1\right) k} I_{i g_{i}} \quad\left(k=0,1, \cdots, g_{i}-1\right)
$$

are linearly independent functions of $I_{i 1}, \ldots, I_{i g_{i}}$. The

$$
g=g_{0}+g_{1}+\cdots+g_{f-1}
$$

characteristic invariants $I_{i j}\left(j=1, \ldots, g_{i} ; i=0,1, \ldots, f-1\right)$ are linearly independent ( $(4)$. Hence the $g$ invariants $\Sigma_{i k}$ are linearly independent. Now $R$ replaces $\Sigma_{i k}$ by $\gamma_{i}^{k} \Sigma_{i k}$. Thus $R^{t}$, of determinant $\rho^{t}$, multiplies $\Sigma_{i k}$ by

$$
\gamma_{i}^{k t}=\left(\rho^{t}\right)^{k e_{i}} .
$$

Hence $\Sigma_{i k}$ is a relative invariant of weight $k e_{i}$.

Let $K=\sum c_{i j} I_{i j}$ be any relative invariant of weight $w$. Under the transformation $R$, of determinant $\rho, K$ becomes $\rho^{n} K$, while $I_{i j}$ becomes $I_{i j-1}$ or $I_{i g_{i}}$ according as $j>1$ or $j=1$. Hence for every $i, j(j>1)$,

$$
c_{i j}=\rho^{i w} c_{i j-1}, \quad c_{i 1}=\rho^{i w} c_{i g_{i}} .
$$

Unless every $c_{i j}=0$, we must have $\rho^{w g_{i}}=1$, so that $w g_{i}$ is a multiple of $p^{n}-1=e_{i} g_{i}$, whence $w=k e_{i}$. In the latter case, $\rho^{w}=\gamma_{i}^{k}$,

$$
\sum_{j=1}^{g_{i}} c_{i j} I_{i j}=c_{i 1} \sum_{j=1}^{g_{i}} \gamma_{i}^{k(j-1)} I_{i j}=c_{i 1} \Sigma_{i k}
$$

Hence $K$ is a linear function of those $\Sigma_{i k}$ which are of weight $w$.

We have now established the following

THEOREM. For a system of s general forms of given degrees in $m$ variables, with arbitrary coefficients in the $G F\left[p^{n}\right]$, the number of linearly independent invariants, absolute and relative, equals the number $g$ of the classes of the systems of forms under the group $G_{1}$ of m-ary linear transformations of determinant unity.

Of the $f$ classes $C_{i}$ under the total m-ary linear group $G$, let the class $C_{i}$ separate into $g_{i}$ classes $C_{i j}$ under $G_{1}$. Let $k$ be any integer such that $0 \leqq k<g_{i}$. Then, for each $i$, there exist $g_{i}$ invariants $\Sigma_{i k}$, given by (2), of weights $k e_{i}$, where* $e_{i}=\left(p^{n}-1\right) / g_{i}$. The $g=g_{q}+\cdots+g_{f-1}$ invariants $\Sigma_{i k}$ are linearly independent. Any invariant of weight $w$ of $G$ is a linear homogeneous function of those of the invariants $\Sigma_{i k}$ which. are of weight $w$.

\footnotetext{
* By introducing notations to indicate which of the numbers $e_{i}$ are equal, we may readily give an explicit formula for the total number of invariants, as well as the number of relative invariants of each weight. For example, the number of absolute invariants is $p^{\text {wy. }}$.
} 
The classes of quadratic forms $q_{m}$ in the $G F\left[p^{n}\right], p>2$.

7. First, let the field be the $G F\left[p^{n}\right], p>2$, and consider the form

$$
q_{m}=\sum_{i, j=1}^{m} \beta_{i v} x_{i} x_{j} \quad\left(\beta_{j i}=\beta_{\mathfrak{V}}\right),
$$

whose coefficients are arbitrary parameters in the field. Then

$$
D=\left|\beta_{i j}\right|
$$

is called the determinant or discriminant of $q_{m}$. For particular values in the field for the coefficients, $q_{m}$ is said to be of rank zero if every $\beta_{i j}=0$, and of rank $r>0$ if at least one minor of order $r$ of $D$ is not zero, while every minor of order greater than $r$ vanishes.

Of various methods leading to a classification of quadratic forms that due to KRONECKER * is best suited for the present application; his theory is seen to hold for any field not having modulus 2 . It is based on two theorems. First, in a symmetrical matrix $\left(\beta_{i j}\right)$ of rank $r>0$, not every principal minor of order $r$ vanishes. Next, if the principal minor

$$
D_{k_{1}, \ldots, k r} \equiv\left|\beta_{k \text { oks }}\right|
$$

$$
(s, t=1, \cdots, r)
$$

is not zero, while every minor of order $>r$ vanishes, there exists a linear transformation of determinant unity which replaces $q_{m}$ by

$$
\sum_{i, t=1}^{r} \beta_{k d k t} x_{k_{0}} x_{k e t} \text {. }
$$

A proof of the last statement will be given in a form convenient for comparison with the treatment in $\S 14$ of the case in which the modulus is 2 . After rearranging the variables, we may assume that, instead of (4),

$$
\left|\beta_{i j}\right| \neq 0
$$$$
(i, j=1, \cdots, r) \text {. }
$$

To $q_{\mathrm{m}}$ we apply the transformation, of determinant unity,

$$
x_{i}=x_{i}^{\prime}+c_{i} x_{m}^{\prime}(i \leqq r), \quad x_{i}=x_{i}^{\prime}(i>r),
$$

and obtain the form

$$
\begin{gathered}
\sum_{i, j=1}^{m-1} \beta_{i j} x_{i}^{\prime} x_{j}^{\prime}+2 \sum_{j=1}^{m-1} B_{j m} x_{j}^{\prime} x_{m}^{\prime}+A x_{m}^{\prime 2}, \\
B_{j m} \equiv \sum_{i=1}^{r} \beta_{i j} c_{i}+\beta_{j m}, \quad A \equiv \sum_{j=1}^{r} B_{j m} c_{j}+B_{m m} .
\end{gathered}
$$

In view of $\left(4^{\prime}\right), c_{1}, \cdots, c_{r}$ can be uniquely determined so that $B_{j m}=0(j \leqq r)$. In the latter and $B_{k m}(r<k \leqq m)$, the determinant of the coefficients of $c_{1}, \ldots, c_{r}, 1$ is the minor of $\beta_{k m}$ in

$$
\left|\beta_{i j}\right| \quad(i, j=1, \cdots, r, k, m) \text {. }
$$

* Werke, vol. 1, p. 166, p. 357. Ct. BôcerE, Introduction to Higher Algebra, p. 58, p. 139 ; GuxDELPiNarr, Journal für reine a. angewand te Mathematik, vol. 91 (1881), p. 221. 
This minor of order $r+1$ is zero by hypothesis. Hence $B_{k m}=0(k>r), A=0$.

Repetitions of the process lead to the form

$$
\sum_{i, j=1}^{r} \beta_{i j} x_{i} x_{j} \text {. }
$$

By a linear transformation of determinant unity, form (5) or (5'), of nonvanishing determinant $\rho$, can be transformed into *

$$
\sum_{i=1}^{r-1} x_{i}^{2}+\rho x_{r}^{2}
$$

while a linear transformation of determinant $\Delta$ replaces $\left(5^{\prime}\right)$ by a form of determinant $\Delta^{2} \rho$. Hence two forms $\left(6_{1}\right)$ and $\left(6_{2}\right)$ are equivalent under the group $G_{1}$ of all $r$-ary linear transformations of determinant unity only when $\rho_{1}=\rho_{2}$; but are equivalent under the total group $G$ if, and only if, $\rho_{1} / \rho_{2}$ is a square in the field.

Under the total group $G$, the class to which the form (6), with $\rho \neq 0$, belongs will be designated by $C_{r, 1}$ or $C_{r,-1}$ according as $\rho$ is a square or a not-square. Thus a complete set of non-equivalent classes of $m$-ary quadratic forms in the $G F\left[p^{n}\right], p>2$, is given by

$$
C_{0}, C_{r, 11} \quad(r=1, \cdots, m) .
$$

Hence a particular form $q_{m}$ of rank $r>0$ belongs to the class $C_{r, 8}$, where $\delta=D_{k_{1}, \ldots, k_{r}}^{\mu}, D_{k_{1}, \ldots, k_{r}}$ being a non-vanishing principal minor of order $r$ of $\left|q_{m}\right|$, and $\mu=\frac{1}{2}\left(p^{n}-1\right)$.

The invariants of a quadratic form in the $G F\left[p^{n}\right], p>2$.

8. We proceed to construct the characteristic absolute invariants $I_{0}, I_{r, \ldots 1}$ of the general quadratic form $q_{m}$ in the $G F\left[p^{n}\right], p>2$, which correspond to the classes (7). By (1),

$$
I_{0}=\Pi\left(1-\beta_{i j}^{2 \mu}\right) \quad(i, j=1, \cdots, m ; i \leqq j) .
$$

Instead of dealing initially with the $I_{r, \ldots 1}$, we first construct an absolute invariant $A_{r}$ which takes the value +1 for the class $C_{r_{1}+1}$, the value -1 for the class $C_{r,-1}$, and the value 0 for the remaining classes $C_{0}, C_{s, \pm 1}(s \neq r)$. Then

$$
I_{r,+1}=\frac{1}{2}\left(A_{r}^{2}+A_{r}\right), \quad I_{r,-1}=\frac{1}{2}\left(A_{r}^{2}-A_{r}\right) \text {. }
$$

For $r=m$, we evidently have

$$
A_{m}=D^{\mu} \quad\left[\mu=\frac{1}{2}\left(p^{n}-1\right)\right] .
$$

For $0<r<m$, the results of $\S 7$ show $(a)$ that $A_{r}=0$ if any principal minor of order $>r$ is not zero; (b) that $A_{r}=0$ if every principal minor of order $\geqq r$ vanishes; and (c) that $A_{r}=M^{\mu}$ if a particular principal minor $M$ of order $r$ is not zero, while every principal minor of order $>r$ vanishes. In view of the

* Dickson, Linear Groups, pp. 157-158. 
first statement $(a)$, we have the identity

$$
A_{r} \equiv \alpha_{r} \Pi\left(1-d^{2 \mu}\right),
$$

where $d$ ranges over the principal minors of order $>r$, while $\alpha_{r}$ is a polynomial in the $\beta_{i j}$ to be determined. Let $M_{1}, \ldots, M_{\rho}$ denote the $\rho=C_{r}^{m}$ principal minors of order $r$, arranged in some definite sequence. In view of the third statement $(c)$,

$$
\alpha_{r}=M_{i}^{\mu} \quad\left(\text { for every } d=0, M_{i} \neq 0\right) .
$$

For $i=1$, the latter yields the identity

$$
\alpha_{r} \equiv M_{1}^{\mu}+K_{1}\left(1-M_{1}^{2 \mu}\right) \quad \text { (for every } d=0 \text { ) } .
$$

To determine $K_{1}$, consider any set of values of the $\beta_{i j}$ which make each $d=0$, $M_{1}=0, M_{2} \neq 0$. Then by (12) for $i=2$, and (13), $M_{2}^{\mu}=K_{1}$. Thus

$$
K_{1} \equiv M_{2}^{\mu}+K_{2}\left(1-M_{2}^{2 \mu}\right) \quad\left(\text { for every } d=0, M_{1}=0\right) .
$$

Then (13) yields * (14) for the case $j=2$ :

$$
\begin{aligned}
\alpha_{r} \equiv & M_{1}^{\mu}+M_{2}^{\mu}\left(1-M_{1}^{2 \mu}\right)+M_{3}^{\mu}\left(1-M_{1}^{2 \mu}\right)\left(1-M_{2}^{2 \mu}\right)+\cdots \\
& \quad+M_{j}^{\mu}\left(1-M_{1}^{2 \mu}\right) \cdots\left(1-M_{j-1}^{2 \mu}\right)+K_{j}\left(1-M_{1}^{2 \mu}\right) \cdots\left(1-M_{j}^{2 \mu}\right),
\end{aligned}
$$

for every set $\beta_{i j}$ such that each $d=0$. For the general step in the derivation of (14), we proceed by induction from $j=t$ to $j=t+1$, assuming that (14) holds for $j=t$. We consider any set $\beta_{i j}$ for which each $d=0, M_{i}=0(i \leqq t)$, $M_{t+1} \neq 0$. Then by (12) for $i=t+1$, and (14) for $j=t, M_{t+1}^{\mu}=K_{t}$. Thus

$$
K_{t}=M_{t+1}^{\mu}+K_{t+1}\left(1-M_{t+1}^{2 \mu}\right)
$$

for all sets $\beta_{i j}$ such that each $d=0, M_{i}=0(i \leqq t)$. Upon substituting this value of $K_{t}$ in (14), for $j=t$, we obtain (14), for $j=t+1$.

Having established (14) for every $j \leqq \rho$, where $\rho$ is the total number of the minors $M$, we consider (14) for $j=\rho$. Let the $\beta_{i}$, have any values such that $M_{i}=0(i \leqq \rho)$ and every $d=0$. By the second statement (b) preceding (11), $A_{r}=0$. Thus, by (14) for $j=\rho, 0=K_{\rho}$. Independently of the restriction that every $d=0,(11)$ and (14) now lead to the result that the explicit expression for the absolute invariant $A_{r}$ is

$$
A_{r}=\left\{M_{1}^{\mu}+M_{2}^{\mu}\left(1-M_{1}^{2 \mu}\right)+\cdots+M_{\rho}^{\mu}\left(1-M_{1}^{2 \mu}\right) \cdots\left(1-M_{p-1}^{2 \mu}\right)\right\} \Pi\left(1-d^{2 \mu}\right),
$$

where $d$ ranges over the principal minors of orders $>r$, while $M_{1}, \ldots, M_{p}$ denote the principal minors of order $r$ taken in any sequence.

The range of $d$ may be restricted to the principal minors of orders $r+1, r+2$.

\footnotetext{
* The restriction $M_{1}=0$ or $K_{1}$ is suppressed in (14) in view of $\left(1-M_{1}^{2 \mu}\right) M_{1}=0$.
} 
We note several forms of invariant (15) when $m=2, r=1$. In that case,

$$
\begin{gathered}
A_{1}=\left(\beta_{22}^{\mu}+\beta_{11}^{\mu}-\beta_{22}^{2 \mu} \beta_{11}^{\mu}\right)\left(1-D^{2 \mu}\right)=\left(\beta_{11}^{\mu}+\beta_{22}^{\mu}-\beta_{11}^{2 \mu} \beta_{22}^{\mu}\right)\left(1-D^{2 \mu}\right), \\
A_{1}=\frac{1}{2}\left(2-\beta_{11}^{\mu} \beta_{22}^{\mu}\right)\left(\beta_{11}^{\mu}+\beta_{22}^{\mu}\right)\left(1-D^{2 \mu}\right) .
\end{gathered}
$$

Applying the useful congruence

$$
(a-b)^{2 \mu} \equiv \sum_{i=0}^{2 \mu} a^{i} b^{2 \mu-i}=\left(a^{\mu}+b^{\mu}\right) \sum_{i=0}^{\mu} a^{i} b^{\mu-i}-a^{\mu} b^{\mu} \quad(\bmod p),
$$

for $a=\beta_{11} \beta_{22}, b=\beta_{12}^{2}$, we get

$$
\begin{aligned}
D^{2 \mu}-1 & =\left(\beta_{11}^{\mu} \beta_{22}^{\mu}+1+\beta_{12}^{2 \mu}-1\right) \sum_{i=1}^{\mu} \beta_{11}^{i} \beta_{22}^{i} \beta_{12}^{2 \mu-2 i}-\beta_{11}^{\mu} \beta_{22}^{\mu} \beta_{12}^{2 \mu}-1 \\
& =\left(\beta_{11}^{\mu} \beta_{22}^{\mu}+1\right)(-1+\Sigma),
\end{aligned}
$$

since $\left(\beta_{12}^{2 \mu}-1\right) \Sigma=\left(\beta_{12}^{2 \mu}-1\right) \beta_{11}^{\mu} \beta_{22}^{\mu}$. Hence $\left(16_{1}\right)$ gives *

$$
A_{1}=\left(\beta_{11}^{\mu}+\beta_{22}^{\mu}\right)\left(1-\sum_{i=0}^{\mu} \beta_{11}^{i} \beta_{22}^{i} \beta_{12}^{2 \mu-2 i}\right) \text {. }
$$

9. Under the group $G_{1}$ of all $m$-ary linear transformations of determinant unity in the $G F\left[p^{n}\right], p>2$, a complete set of non-equivalent classes of $m$-ary quadratic forms is given by

$$
C_{0}, C_{r, \pm 1}(r=1, \cdots, m-1), C_{m, p}(\rho \text { arbitrary } \neq 0),
$$

where $C_{m, \rho}$ is the class containing (6) for $r=m$. In that case, different $\rho$ 's give non-equivalent forms under $G_{1}(\S 7)$. But for $r<m,(6)$ is transformed into \& like form, with the parameter $\rho a^{2}$, by the transformation

$$
x_{r}=a x_{r}^{\prime}, \quad x_{m}=a^{-1} x_{m}^{\prime}, \quad x_{i}=x_{i}^{\prime} \quad(i+r, m)
$$

of determinant unity. Let $\phi$ be a relative invariant of weight 20 of $q_{m}$, so that $\phi$ becomes $\Delta^{\infty} \phi$ under a transformation of determinant $\Delta$. But each class is transformed into itself by every transformation of determinant \pm 1 (in particular, by the one changing the sign of $x_{1}$ ). Hence $w$ is even (cf. $\S 6$ ).

Of the classes (7) under the total group $G$, classes $C_{m, \pm 1}$ alone separate into subclasses $C_{m, p}$ under $G_{1}$. If $\phi$ has the value $v$ for a class $C_{m, p}$, then $\phi$ has the value $\Delta^{* v} v$ for the class $C_{m, p}$ obtained from $C_{m, \rho}$ by a transformation of determinant $\Delta$. Hence, if $\rho^{\prime} / \rho$ is a square, the value of $\phi$ for $C_{m, \rho^{\prime}}$ equals $\left(\rho^{\prime} / \rho\right)^{m / 2}$ times its value for $C_{m, \rho}$. Thus arbitrary values can be assigned to $\phi$ for just one of the $\mu=\frac{1}{2}\left(p^{n}-1\right)$ classes $C_{m, p}$ in which $\rho$ is a square, and for just one of the $\mu$ classes $C_{m, \rho}$ in which $\rho$ is a not-square. When these two assigned values are zero, the invariant is absolute and has been constructed in $\S 8$. The

\footnotetext{
* Invariant (19) is the negative of $Q$, these Transacti ons, vol. 8 (1907), pp. 211, 217, 218.
} 
same is true if a non-vanishing value be assigned to $\phi$ for one of the classes $C_{0}, C_{r, \pm 1}(r<m)$. Hence to obtain a non-absolute invariant $\phi$ of weight $2 \delta$, where $0<2 \delta<p^{n}-1$, we must assign to $\phi$ the value zero for $C_{0}, C_{r, \pm 1}(r<m)$, and values not both zero for two classes $C_{m, p}$, in one of which $\rho$ is a particular square, in the other $\rho$ is a particular not-square. Since the values of $\phi$ for every class are then uniquely determined, an unique polynomial $\phi$ can be constructed $(\S 1)$. Hence there are exactly two linearly independent invariants of weight $2 \delta$. But, if $D$ is the determinant of $q_{m}$, then $D^{\delta}$ and $D^{\delta+\mu}$ are linearly independent invariants of weight $2 \delta$.

Combining the present results with those in $\S 8$, we obtain the

Theorem. A complete set of linearly independent invariants of the m-ary quadratic form in the $G F\left[p^{n}\right], p>2$, is given by the $2 m+1$ characteristic absolute invariants $I_{0}, I_{r, \pm 1}(r=1, \ldots, m)$, and the $p^{n}-3$ relative invariants $D^{\delta}, D^{\delta+\mu}(\delta=1, \cdots, \mu-1)$, where $\mu=\frac{1}{2}\left(p^{n}-1\right)$. As an alternative set, we may take

$$
I_{0}, A_{r}, A_{r}^{2}(r=1, \ldots, m-1), \quad D^{k}\left(k=1, \cdots, p^{n}-1\right) .
$$

The invariants mentioned are defined by $\left(3^{\prime}\right),(8),(9),(10),(15)$.

The product of any two invariants of the first set can be reduced to a linear combination of those in the first set by means of the relations *

$$
\begin{aligned}
D^{2 \mu+1} & =D, \quad I_{0} D=I_{r, \pm 1} D=0(r<m), \quad I_{m, \pm 1} D=\frac{1}{2}\left(D \pm D^{\mu+1}\right), \\
I^{\frac{1}{2}} & =I, \quad I I^{\prime}=0\left(I \text { and } I^{\prime} \text { any pair of the } I_{0}, I_{r, \pm 1}\right) .
\end{aligned}
$$

For the set (21), the following relations suffice :

$$
D^{2_{\mu+1}}=D, I_{0}^{2}=I_{0}, A_{r}^{3}=A_{r}, I_{0} A_{r}=I_{0} D=A_{r} D=A_{r} A_{c}=0(r \neq 8) \text {. }
$$

Reduction of quadratic forms in the GF $\left[2^{n}\right], \S \S 10-15$.

10. In view of the application to be made for fields having the modulus 2 , we consider some algebraic properties of the quadratic form

$$
Q_{m}=\sum \beta_{i j} x_{i} x_{j}+\sum b_{j} x_{j}^{2} \quad(i, j=1, \cdots, m ; i<j) .
$$

Its discriminant $D$ and a related skew-symmetric determinant $d$ are

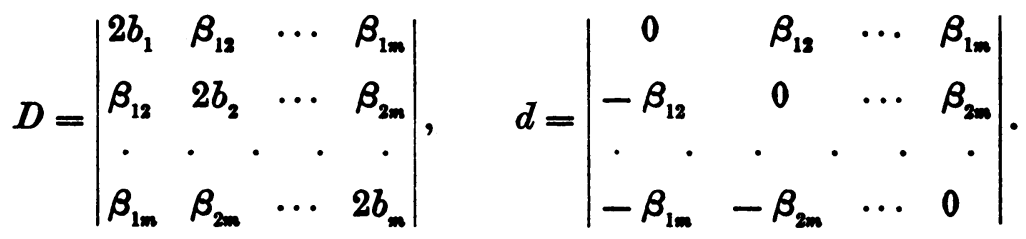

Now $d \equiv 0$ for $m$ odd; while, for $m$ even, $d$ equals the square of the pfaffian $\dagger$

* These follow at once from the values of the invariants for the various classes.

† We consider piaffians in the $\beta_{v}$ exclusively. Note that [12] denotes $\beta_{19}$. 
[12 $\cdots m]$. Since corresponding elements of $D$ and $d$ differ by multiples of 2,

$$
D=2 S_{m} \quad(m \text { odd }), \quad D=[12 \cdots m]^{2}+2 R \quad(m \text { even }) \text {. }
$$

If $Q_{m}$ becomes $Q_{m}^{\prime}$ under a linear homogeneous transformation of determinant $\Delta$, the discriminant $D^{\prime}$ of $Q_{m}^{\prime}$ equals $\Delta^{2} D$. Hence, for $m$ odd, $S_{m}$ is invariant up to the factor $\Delta^{2}$; while, for $m$ even,

$$
[12 \cdots m]_{\beta^{\prime}}=\Delta[12 \cdots m]_{\beta}+2 \rho,
$$

since the $\beta_{i j}^{\prime}$ involve only even multiples of the $b_{k}$.

11. Let the coefficients of $Q_{m}$ belong to the $G F\left[2^{n}\right]$. If any coefficient be increased by a multiple of $2, D$ is increased by a multiple of 4 ; thus $S_{m}$ and $[12 \ldots m]$ are increased by multiples of 2 . It will prove convenient to employ the notation $\{12 \cdots m\}$ for $S_{m}$. According as $m$ is even or odd, [12 $\left.\cdots m\right]$ or $\{12 \ldots m\}$ is an invariant * of $(24)$ in the $G F\left[2^{n}\right]$.

We shall consider two methods of normalizing $Q_{m}$ under linear transformation in the $G F\left[2^{n}\right]$, each possessing certain advantages. The contrast between the two methods is analogous to that between the methods of LAGRANGe and KRONECKER in the algebraic theory. The present theory is essentially different from the algebraic theory; this is due partly to the fact that the terms involving the squares of the variables hold themselves aloof under transformation.

12. If every $\beta_{i j}=0, Q_{m}$ is the square of $\sum b_{j}^{j} x_{j}$ in the $G F\left[2^{n}\right]$ and either vanishes identically or can be transformed into $x_{1}^{2}$. If not every $\beta_{i}$ vanishes, let $\beta_{12} \neq 0, m>2$. Under the transformation

$$
x_{1}^{\prime}=x_{1}+\sum_{i=3}^{m} \beta_{2 i} x_{i}, \quad x_{2}^{\prime}=x_{2}+\sum_{i=3}^{m} \beta_{1 i} x_{i}, \quad x_{j}^{\prime}=\beta_{12} x_{j} \quad(j=3, \cdots, m),
$$

of determinant $\beta_{12}^{m-2}, Q_{m}\left(x^{\prime}\right)$ becomes $\dagger$

$$
\beta_{12} x_{1} x_{2}+\beta_{12} \Sigma[12 i j] x_{i} x_{j}+b_{1} x_{1}^{2}+b_{2} x_{2}^{2}+\sum\{12 i\} x_{i}^{2} \quad(i, j=3, \cdots, m ; i<j),
$$

where $[12 i j]$ is a pfaffian, while

$$
\{12 i\}=\beta_{12} \beta_{1 i} \beta_{2 i}+b_{1} \beta_{2 i}^{2}+b_{2} \beta_{1 i}^{2}+b_{i} \beta_{12}^{2} \text {, }
$$

so that $\{123\}$ is the semi-discriminant of $Q_{3}$. Hence, for $m=3$ or 4 , the vanishing of the invariant $\{123\}$ or $\{1234\}$ is a necessary and sufficient condition that $Q_{3}$ or $Q_{4}$, with $\beta_{12} \neq 0$, shall be tranformable into a binary or ternary form, respectively.

Next, let $m>4$. If every $[12 i j]=0,(27)$ can be transformed into a ternary form. In the contrary case, let $[1234] \neq 0$ and apply the following discussion for $l=2$, with $[1 \ldots 0]$ replaced by unity, $\{1 \ldots 0 k\}$ replaced by $b_{k}$.

* Amerioan Journal of Mathematios, vol. 30 (1908), p. 265.

†Ibid., p. 264, formula (2), with $x_{2}$ replaced by $c_{12} x_{2}$. 
The general step in the reduction process will be made by induction. Let $l$ be an integer $\leqq m / 2$ such that not every pfaffian of order $2 l$ vanishes; in particular, let

$$
[12] \neq 0,[1234] \neq 0, \cdots,[1 \cdots 2 l] \neq 0 .
$$

We assume that, after $l-1$ steps, $Q_{m}$ has been transformed into

$$
[12] x_{1} x_{2}+[12][1234] x_{3} x_{4}+[1234][1 \cdots 6] x_{5} x_{6}+\cdots
$$

$$
\begin{aligned}
& +[1 \cdots 2 l-4][1 \cdots 2 l-2] x_{2 l-3} x_{2 l-2}+[1 \cdots 2 l-2] \sum[1 \cdots 2 l-2 i j] x_{i} x_{j} \\
& +b_{1} x_{1}^{2}+b_{2} x_{2}^{2}+\{123\} x_{3}^{2}+\{124\} x_{4}^{2}+\cdots+\{1 \cdots 2 l-42 l-3\} x_{2 l-3}^{2} \\
& +\{1 \cdots 2 l-42 l-2\} x_{2 l-2}^{2}+\sum\{1 \cdots 2 l-2 i\} x_{i}^{2}-(i, j=2 l-1, \cdots, m ; i<j),
\end{aligned}
$$

by a transformation of determinant

$$
[12]^{2}[1234]^{2} \cdots[1 \ldots 2 l-4]^{2}[1 \cdots 2 l-2]^{m-2 l+2} \text {. }
$$

To (29), with each $x$ accented, we.apply the transformation

$$
\begin{array}{r}
x_{2 l-1}^{\prime}=x_{2 l-1}+t \sum_{i=2 l+1}^{m}[1 \ldots 2 l-22 l i] x_{i}, \quad x_{2 l}^{\prime}=x_{2 l}+t \sum_{i=2 l+1}^{m}[1 \ldots 2 l-22 l-1 i] x_{i}, \\
x_{j}^{\prime}=t[1 \ldots 2 l] x_{j} \quad(j=2 l+1, \cdots, m),
\end{array}
$$

where $t=[1 \cdots 2 l-2]^{-1}$. This alters only the terms of (29) under the two summation signs. These terms are replaced by

$$
\begin{aligned}
& {[1 \cdots 2 l-2][1 \cdots 2 l] x_{2 l-1} x_{2 l}+[1 \cdots 2 l] \sum \sigma_{i j} x_{i} x_{j}} \\
& +\{1 \cdots 2 l-22 l-1\} x_{2 l-1}^{2}+\{1 \cdots 2 l-22 l\} x_{2 l}^{2}+\sum f_{i} x_{i}^{2} \\
& \quad \cdot(i, j=2 l+1, \cdots, m ; i<j),
\end{aligned}
$$

where

$$
\begin{aligned}
\sigma_{i j}= & t\{[1 \ldots 2 l-22 l i][1 \ldots 2 l-22 l-1 j] \\
& +[1 \ldots 2 l-22 l j][1 \ldots 2 l-22 l-1 i]+[1 \ldots 2 l][1 \ldots 2 l-2 i j]\}, \\
f_{i}= & t^{2}\left([1 \ldots 2 l-22 l i]^{2}\{1 \ldots 2 l-22 l-1\}\right. \\
& +[1 \ldots 2 l-22 l-1 i]^{2}\{1 \ldots 2 l-22 l\}+[1 \ldots 2 l]^{2}\{1 \ldots 2 l-2 i\} \\
& \quad+[1 \ldots 2 l-2][1 \ldots 2 l][1 \ldots 2 l-22 l-1 i][1 \ldots 2 l-22 l i]) .
\end{aligned}
$$

Hence the induction will be complete if it is shown that

$$
\sigma_{i j}=[1 \ldots 2 l i j], \quad f_{i}=\{1 \ldots 2 l i\} .
$$

The product of the determinant of (31) by (30) is clearly

$$
[12]^{2}[1234]^{2} \cdots[1 \ldots 2 l-2]^{2}[1 \ldots 2 l]^{m-2 l} \text {. }
$$

Although $\left(34_{1}\right)$ may be established by means of the algebraic theory of 
pfaffians, we shall prove both parts of (34) by applying the invariance of the discriminant or semi-discriminant, up to a factor $\Delta^{2}$, under a transformation of determinant $\Delta$ in the $G F\left[2^{n}\right]$. We note that the preceding development is valid for any $m \geqq 2 l$. First, we take $m=2 l+2$. Then the discriminant of the final quadratic form is seen to equal $B^{2} \sigma_{2 l+12 l+2}^{2}$, where $B$ is the product (35), the final exponent being 2. But the discriminant of $Q_{2 l+2}$ is $[1 \cdots 2 l+2]^{2}$. Hence $\left(34_{1}\right)$ is true for $i=2 l+1, j=2 l+2$, and therefore for any $i, j$ exceeding $2 l$. Next for $m=2 l+1$, the semi-discriminant of the final form equals $P^{2} f_{2 l+1}$, where $P$ is the product (35) with the last exponent unity. But the semi-discriminant of $Q_{2 l+1}$ is $\{1 \ldots 2 l+1\}$. Hence $\left(34_{2}\right)$ holds for $i=2 l+1$, and therefore for every $i>2 l$.

We make the further assumption that every pfaffian of order $>2 l$ vanishes. The form reached above thus becomes

$$
\begin{aligned}
\sum_{s=1}^{l}[1 \cdots 2 s-2][1 \ldots 2 s] x_{2 s-1} x_{24}+\sum_{i=2 l+1}^{m}\{1 \cdots 2 l i\} x_{i}^{2} \\
\quad+\sum_{s=1}^{l}\{1 \cdots 2 s-22 s-1\} x_{2 s-1}^{2}+\sum_{s=1}^{l}\{1 \ldots 2 s-22 s\} x_{2 s}^{2}-
\end{aligned}
$$

To this form we apply the transformation

$x_{2 s}=[1 \ldots 2 s]^{-1} x_{2 s}^{\prime}, \quad x_{2 s-1}=[1 \cdots 2 s-2]^{-1} x_{2 s-1}^{\prime}(s \leqq l), \quad x_{i}=x_{i}^{\prime}(i>2 l)$, and then drop the accents on the $x^{\prime}$. We get

$$
\begin{aligned}
\sum_{s=1}^{l} x_{2 s-1} x_{2 s}+\sum_{i=1}^{2 l} \delta_{i} x_{i}^{2}+\sum_{s=2 l+1}^{m}\{1 \ldots 2 l i\} x_{i}^{2}, \\
\delta_{2 s-1}=[1 \ldots 2 s-2]^{-2}\{1 \ldots 2 s-22 s-1\}, \quad(s=1, \ldots, l) \\
\delta_{2 s}=[1 \ldots 2 s]^{-2}\{1 \ldots 2 s-22 s\}
\end{aligned}
$$

The product of the determinant of this transformation by (35) is

$$
[1 \ldots 2 l]^{m-2 l-1}
$$

Hence $Q_{m}$ is transformed into (37) by a transformation of determinant (39).

First, let at least one of the $\{1 \ldots 2 l i\}$ be not zero, where $i>2 l$, thus. implying that $m>2 l$. Then, by (37), $Q_{m}$ can be transformed into

$$
\sum_{d=1}^{l} x_{2 o-1} x_{2 t}+c x_{2 l+1}^{2} \quad(c=1 \text { if } m>2 l+1, c=\{1 \cdots 2 l+1\} \text { if } m=2 l+1) \text {. }
$$

by a transformation of determinant unity. Under the group of transformations of all determinants, (40) with $m=2 l+1$ is equivalent to a like form with $c=1$. In view of the invariance of the semi-discriminant, $(40)$ can not be transformed into a form on fewer than $2 l+1$ variables. Now $\{1 \ldots 2 l i\} \neq 0$ 
implies, in view of (33) and (34), that not every pfaffian of order $2 l$ is zero. Hence necessary and sufficient conditions that $Q_{m}$ shall be transformable into a form on $2 l+1$ variables, but not into a form on $2 l$ variables, are that every pfaffian of order $>2 l$ vanishes and that not every principal semi-minor $\left\{i_{1} i_{2} \cdots i_{2 l+1}\right\}$ of order $2 l+1$ varishes.

Next, let every $\{1 \cdots 2 l i\}=0$. Then by a linear transformation of determinant unity, (37) can be transformed * into

$$
\sum_{s=1}^{l} x_{2 s-1} x_{2 s}+x_{1}^{2}+\delta x_{2}^{2}, \quad \delta \equiv \sum_{s=1}^{l} \delta_{2 t-1} \delta_{2 s} .
$$

Now $x_{1} x_{2}+x_{1}^{2}+\delta x_{2}^{2}$ is reducible or irreducible in the $G F\left[2^{n}\right]$ according as $\chi(\delta)=0$ or 1 , where

$$
\chi(\delta)=\sum_{i=0}^{n-1} \delta^{2^{i}} \quad \chi^{2} \equiv \chi .
$$

The forms (41) with $\chi(\delta)=0$ are all equivalent to

$$
\sum_{s=1}^{l} x_{2 o-1} x_{2 s}
$$

under the group $G_{1}$ of linear transformations of determinant unity. The forms (41) with $\chi(\delta)=1$ are equivalent under $G_{1}$, but no one of them can be transformed into (43). The reduction of $Q_{m}$ to (37) was effected by a transformation of determinant (39). If $m>2 l, Q_{m}$ can be transformed into (41) within $G_{1}$; but if $m=\boldsymbol{\imath}$, the canonical form within $G_{1}$ may be taken to be (41) with one of the variables multiplied by $[1 \ldots 2 l]$. Among the results established, we mention the following:

Necessary and sufficient conditions that $Q_{m}$ shall be transformable into a form on $2 l$ variables, but not into a form on $2 l-1$ variables, are that every pfaffian of order $>2 l$ and every $\left\{i_{1} \cdots i_{2 l+1}\right\}$ shall vanish, but not every pfaffian of order $2 l$.

13. A quadratic form in the $G F\left[2^{n}\right]$ may be said to be of rank $r$ if, of the principal minors of even order and the algebraic halves of the principal minors of odd order, those of order $>r$ vanish, but not all of order $r$ vanish. As an equivalent definition we may say that $Q_{m}$ is of odd rank $2 l+1$ if every $\dagger$ $\left[i_{1} \cdots i_{2 l+2}\right]$ but not every $\left\{i_{1} \cdots i_{2 l+1}\right\}$ vanishes; that $Q_{m}$ is of even rank $2 l$ if every $\left[i_{1} \cdots i_{2 l+2}\right]$ and every $\ddagger\left\{i_{1} \cdots i_{2 l+1}\right\}$ but not every $\left[i_{1} \cdots i_{2 l}\right]$ vanishes.

In comparing these definitions with the algebraic definition of the rank of a quadratic form or symmetrical matrix, we note that a principal minor of odd

* Amerioan Journal of Mathematios, vol. 30 (1908), p. 266, \$6.

† Then every pfaffian of order $>2 l$ vanishes, also every $\left\{i_{1} \cdots i_{t}\right\}, t>2 l+2$, by (33), (34).

$\ddagger$ The vanishing of these does not imply that of the pfaffians of order $\geqq 2 l+2$, as shown by the example $x_{1} x_{2}+x_{3} x_{4}+\cdots+x_{2 l+1} x_{2 l+2}$. 
order vanishes identically modulo 2 , so that we have introduced their algebraic halves; furthermore, when a principal minor $M$ of even order vanishes in the $G F\left[2^{n}\right]$, all the first minors * $M_{i j}$ of $M$ also vanish, since $M_{i i} M_{j j}-M_{i j}^{2}$ is a multiple of $M$ and since $M_{i i}$ is multiple of 2 .

From the results stated in italics in $\$ 12$ we have the

THEOREM. A quadratic form in the $G F\left[2^{n}\right]$ can be transformed into a form on $r$ variables, but not into one on $r-1$ variables, if and only if its rank is $r$.

Second method of reduction of a quadratic form.

14. We consider a second method $\dagger$ of normalizing a quadratic form (24) in the $G F\left[2^{n}\right]$. If $[1 \cdots m] \neq 0$, so that $m$ is an even number $2 l, Q_{m}$ is already of the form (50). In the contrary case there is some integer $l<m / 2$ such that every pfaffian of order $>2 l$, but not every pfaffian of order $2 l$, vanishes. We assume that $l>0$, thus excluding the rather trivial case in which every $\beta_{i j}=0$. After rearranging the variables, we may set $[1 \ldots 2 l] \neq 0$. To $Q_{m}$ we apply the transformation

$$
x_{i}=x_{i}^{\prime}+c_{i} x_{m}^{\prime}(i=1, \ldots, 2 l), \quad x_{i}=x_{i}^{\prime}(i=2 l+1, \ldots, m) .
$$

Let $\beta_{i i}=\beta_{i j}, \beta_{i i}=0$. Then the resulting form is

$$
\begin{gathered}
\sum_{i<j}^{1, \ldots-1} \beta_{i j} x_{i}^{\prime} x_{j}^{\prime}+\sum_{j=1}^{m-1} B_{j m} x_{j}^{\prime} x_{m}^{\prime}+\sum_{i=1}^{m-1} b_{i} x_{i}^{\prime 2}+E_{m} x_{m}^{\prime 2}, \\
B_{j m}=\sum_{i=1}^{2 l} \beta_{i j} c_{i}+\beta_{j m}, \quad E_{m}=\sum_{j=1}^{2 l} \beta_{j m} c_{j}+{ }^{1, \ldots \sum^{2 l}} \beta_{i j} c_{i} c_{j}+\sum_{i=1}^{2 l} b_{i} c_{i}^{2}+b_{m} .
\end{gathered}
$$

By choice of the $c_{i}$, we may make $B_{j m}=0(j=1, \ldots, 2 l)$. In fact, $[1 \ldots 2 l]^{2} c_{i}$ equals the minor of $\beta_{\text {im }}$ in the skew-symmetric determinant giving the square of $[1 \ldots 2 l m]$. Apart from sign, this minor equals $\ddagger$

Hence

$$
[1 \ldots i-1 i+1 \ldots 2 l m][1 \ldots 2 l] \text {. }
$$

$$
c_{i}=[1 \ldots 2 l]^{-1}[1 \ldots i-1 i+1 \ldots 2 l m] \quad(i=1, \ldots, 2 l) .
$$

For these values of the $c_{i}$, we have $B_{j m}=0$ for any $j$, as may be shown from (46) or without computation as follows. The determinant of the coefficients of

* As shown earlier, the introduction of the $\frac{1}{2} M_{i l}$ serves to define the rank. Contrary to the suggestions in my earlier papers I now prefer to avoid the use of semi-minore other than prinoipal, since $\frac{1}{2} M_{U}(i+j)$ is defined in the field only when $M=0$, and even then in a very artificial manner.

† Having points of resemblance and points of contrast with KRONECKER's algebraio method (\&7).

$\ddagger$ ScoT, Theory of Determinants, 1880, p. 75, \$15. 
$c_{1}, \cdots, c_{2 l}, 1$ in $B_{j m}(j=1, \ldots, 2 l, k)$ is the minor $M_{k m}$ of the element $\beta_{k m}$ in the last row of

$$
D_{2 l+2}=\left|\beta_{a t}\right| \quad(s, t=1, \cdots, 2 l, k, m) .
$$

The latter equals $[1 \ldots 2 l \mathrm{~km}]^{2}$ and is zero by hypothesis. Let $M_{i i}$ denote the minor of $\beta_{i i}$, so that $M_{i i}=0$. But $M_{k k} M_{m m}-M_{k m}^{2}$ equals the product of $D_{2 l+2}$ by the minor $\left|\beta_{a t}\right|, s, t=1, \ldots, 2 l$. Hence $M_{k m}=0$.

To evaluate $E_{m}$, we note that the preceding discussion is valid for any $m$. For $m=2 l+1$, the semi-discriminant of (44), with each $B_{j m}=0$, equals $[1 \ldots 2 l]^{2} E_{2 l+1}$, while that of $Q_{2 l+1}$ is $\{1 \ldots 2 l+1\}$. Now the first sum in $E_{m}$, given by (45), becomes a multiple of 2 when $\beta_{j m}$ is eliminated by means of $B_{j m}=0$. Hence

$$
\{1 \ldots 2 l+1\}=[1 \ldots 2 l]^{2}\left\{\sum_{i<j}^{1, \ldots 2 l} \beta_{i j} c_{i} c_{i}+\sum_{i=1}^{2 l} b_{i} c_{i}^{2}+b_{2 l+1}\right\}
$$

becomes an identity when the values (46), with $m=2 l+1$, are inserted :

$$
\begin{aligned}
& \{1 \cdots 2 l+1\}=\sum_{i<i}^{1, \ldots 2 l} \beta_{i j}[1 \cdots i-1 i+1 \cdots 2 l+1] \\
& \quad \times[1 \cdots j-1 j+1 \cdots 2 l+1]+\sum_{i=1}^{2 l+1} b_{i}[1 \cdots i-1 i+1 \cdots 2 l+1]^{2} .
\end{aligned}
$$

Replacing the subscript $2 l+1$ by $m$, we derive the identity

$$
\{1 \ldots 2 l m\} \equiv[1 \ldots 2 l]^{2} E_{m} .
$$

For the next step, we add suitable multiples of $x_{m-1}$ to $x_{1}, \cdots, x_{21}$. After $m-2 l$ such steps, we find that $Q_{m}$ has been reduced, by a transformation of determinant unity, to

$$
\sum_{i<j}^{1, \ldots, 2 l} \beta_{i j} x_{i} x_{j}+\sum_{i=1}^{2 l} b_{i} x_{i}^{2}+[1 \ldots 2 l]^{-2} \sum_{i=2 l+1}^{m}\{1 \ldots 2 l i\} x_{i}^{2} .
$$

The first two sums define $Q_{2 l}$, viz., (24) for $m=2 l$.

15. For the further normalization of (50) we shall apply transformations involving only the variables $x_{1}, \cdots, x_{2 l}$. In particular, the final sum in (50) will not be altered. The present problem is therefore the normalization of $Q_{2 t}$ of discriminant $[1 \ldots 2 l]^{2} \neq 0$. Thus not every pfaffian of order $2 l-2$ vanishes; let $[1 \ldots 2 l-2] \neq 0$. We may proceed as in $\$ 14$ (with $m$ replaced by $2 l$, and $l$ by $l-1$ ) with a certain essential modification.* The transformation

$x_{i}=x_{i}^{\prime}+[1 \ldots 2 l-2]^{-1}[1 \ldots i-1 i+1 \ldots 2 l-22 l] x_{3 l}^{\prime}(i=1, \ldots, 2 l-2)$,

$$
x_{2 l-1}=x_{2 l-1}^{\prime}, \quad x_{2 i}=x_{2 l}^{\prime},
$$

* In the proof that $B_{j m}=0(j>2 l)$ by means of the vanishing of the plaffians of order $2+2$. The latter correspond to plafians of order $2 l$ in the present case, and these are not all zero. Instead of the $B_{f_{m}}$ wo now have $B_{l}$, which does not vanish. 
of determinant unity, replaces $Q_{2 l}$ by

$$
\sum_{i<j}^{1, \ldots, 2 l-1} \beta_{i j} x_{i}^{\prime} x_{j}^{\prime}+B_{i} x_{2 l-1}^{\prime} x_{2 l}^{\prime}+\sum_{i=1}^{2 l-1} b_{i} x_{i}^{\prime 2}+[1 \ldots 2 l-2]^{-2}\{1 \ldots 2 l-22 l\} x_{2 l}^{\prime 2},
$$

where

$$
\begin{aligned}
B_{l} \equiv \sum_{i=1}^{2 l-2} \beta_{i 2 l-1}[1 \ldots 2 l-2]^{-1}[1 \ldots i-1 i & +1 \ldots 2 l-22 l]-\beta_{2 l-1} 2 l \\
& =[1 \ldots 2 l-2]^{-1}[1 \ldots 2 l] .
\end{aligned}
$$

The last equality follows from the expansion of $[1 \ldots 2 l]$ with respect to the elements $\beta_{i 2 l_{-1}}$, or by the identity of the discriminants of $Q_{22}$ and (51).

Similarly, to (51) we apply the transformation

$$
\begin{gathered}
x_{i}^{\prime}=x_{i}^{\prime \prime}+[1 \ldots 2 l-2]^{-1}[1 \ldots i-1 i+1 \cdots 2 l-22 l-1] x_{2 l-1}^{\prime \prime} \quad(i=1, \cdots, 2 l-2), \\
x_{2 l-1}^{\prime}=x_{2 l-1}^{\prime \prime}, \quad x_{2 l}^{\prime}=x_{2 l}^{\prime \prime},
\end{gathered}
$$

suppress the accents on $x^{\prime \prime}$, and obtain the form

$$
\begin{aligned}
& \sum_{i<j}^{1, \ldots, 2 l} \beta_{i j} x_{i} x_{j}+[1 \ldots 2 l-2]^{-1}[1 \ldots 2 l] x_{2 l-1} x_{2 l}+\sum_{i=1}^{2 l-2} b_{i} x_{i}^{2} \\
& \quad+[1 \ldots 2 l-2]^{-2}\{1 \ldots 2 l-22 l-1\} x_{2 l-1}^{2}+[1 \ldots 2 l-2]^{-2}\{1 \ldots 2 l-22 l\} x_{2 l}^{2} .
\end{aligned}
$$

The first and third sums define $Q_{2 l-2}$. The double step by which $Q_{21}$ has been reduced to (52) by a transformation of determinant unity may now be repeated. By rearranging the variables $x_{1}, \ldots, x_{21-2}$, we may assume that (28) holds. We arrive ultimately at the following conclusion: If every pfaffian of order $>2 l$, but not every pfaffian of order $2 l$, vanishes, the pfaffians [12], $[1234], \ldots,[1 \ldots 2 l]$ may be assumed not to vanish ; then $Q_{m}$ may be reduced by a linear transformation of determinant unity to

$$
\begin{aligned}
\sum_{s=1}^{l}[1 \ldots 2 s-2]^{-1}[1 \ldots 2 s] x_{2 s-1} x_{2 s}+\sum_{s=1}^{l}[1 \ldots 2 s-2]^{-2}\left(\{1 \ldots 2 s-22 s-1\} x_{20-2}^{2}\right. \\
\left.+\{1 \ldots 2 s-22 s\} x_{2 s}^{2}\right)+[1 \ldots 2 l]^{-2} \sum_{s=2 l+1}^{m}\{1 \ldots 2 l i\} x_{i}^{2} .
\end{aligned}
$$

If we multiply $x_{2,-1}$ and $x_{2 t}$ by $[1 \ldots 2 s-2]$, for $s=1, \ldots, l$; and $x_{i}$ by $[1 \ldots 2 l]$, for $i=2 l+1, \ldots, m$, we see that $Q_{m}$ can be reduced by a linear transformation of determinant (35) to the form (36).

Hence the present method of reduction has led us to the same normal form as that obtained by the former method.

Definition and construction of the invariant $\chi_{2}$.

16. It remains to investigate a problem of decided importance both for the general theory of the reduction of quadratic forms in the $G F\left[2^{n}\right]$ and for the 
subsequent determination of the invariants. Under the assumption (28) that no one of the pfaffians [12], [1234], .., [1 ..2l] vanishes, the form $Q_{2 l}$ was transformed into the normal form (41), in which

$$
\delta=\sum_{s=1}^{l}[1 \ldots 2 s-2]^{-2}[1 \ldots 2 s]^{-2}\{1 \ldots 2 s-22 s-1\}\{1 \ldots 2 s-22 s\} .
$$

Of the forms (41), there are two non-equivalent canonical forms distinguished by the value 0 or 1 of $\chi(\delta)$, where $\chi$ is the function (42). Given a form $Q_{2 l}$ having $[1 \ldots 2 l] \neq 0$, we readily obtain by a suitable rearrangement of the variables a form $Q_{2 l}^{\prime}$ for which the assumption (28) holds, so that the criterion $\chi(\delta)=0$ or 1 is applicable. What we desire, however, is a criterion which will apply directly to $Q_{22}$ itself. Moreover, we prefer to accomplish this result by means of an absolute invariant* of $Q_{2 l}$, which must therefore be defined for all values of the coefficients including those making $[1 \ldots 2 l]=0$. Hence we seek a polynominal $\chi_{l}$ in the coefficients of $Q_{2 l}$ such that $\chi_{l}=1$ when $Q_{2 l}$ is transformable into (41) with $\chi(\delta)=1$, while $\chi_{t}=0$ for all remaining forms $Q_{21}$. Such a polynominal $\chi_{l}$ is clearly an absolute invariant of $Q_{2 l}$.

For $l=1, \beta_{12} \neq 0,(54)$ gives $\delta=b_{1} b_{2} \beta_{12}^{-2}$. Hence we have

$$
\chi_{1}=\chi\left(b_{1} b_{2} \beta_{12}^{2 n-3}\right) \text {, }
$$

where (and below) an exponent $2^{n}-3$ is to be replaced by unity when $n=1$.

Next, let $l=2$. For $\beta_{12} \neq 0, P \equiv[1234] \neq 0,(54)$ gives

$$
\delta=\beta_{12}^{-2} b_{1} b_{2}+\beta_{12}^{-2} P^{-2}\{123\}\{124\} \text {. }
$$

Thus $\delta=\delta_{12}$, where

$$
\delta_{i j}=\left(b_{i} b_{j} P^{2}+\{i j k\}\{i j t\}\right)\left(\beta_{i j} P\right)^{2 n-3},
$$

$i, j, k, t$ being a permutation of $1,2,3,4$. Hence

$$
\chi_{2}=\chi\left(\delta_{i}\right)
$$

for every set of values of the 10 coefficients of $Q_{4}$ for which $\beta_{i} \neq 0$ (whether or not $P \neq 0$ ). In particular, we deduce the identity

$$
\chi_{2} \equiv \chi\left(\delta_{12}\right)+M\left(\beta_{12}^{\nu}-1\right) \quad\left(\nu=2^{n}-1\right) .
$$

Consider any set with $\beta_{12}=0, \beta_{13} \neq 0$. By (58), and (57) for $i, j=1,3$,

$$
\begin{aligned}
& {\left[\chi\left(\delta_{13}\right)\right]_{\beta_{13}=0}=M} \\
& \left(\text { for } \beta_{13}+0\right) \\
& \equiv M+M_{1}\left(\beta_{13}^{\nu}-1\right) \text {, }
\end{aligned}
$$

where $M_{1}$ is free of $\beta_{12}, \beta_{13}$. Then (58) becomes

$$
\chi_{2} \equiv \chi\left(\delta_{12}\right)+\left(\beta_{12}^{v}-1\right) \chi\left(\delta_{13}\right)+M_{1}\left(\beta_{12}^{v}-1\right)\left(\beta_{13}^{v}-1\right) .
$$

* For the GF $\left[p^{n}\right], p>2$, we used the power $\frac{1}{2}\left(p^{n}-1\right)$ of the discriminant. 
Consider any set with $\beta_{12}=\beta_{13}=0, \beta_{14} \neq 0$. By (59) and (57) for $i, j=1,4$,

$$
\begin{aligned}
{\left[\chi\left(\delta_{14}\right)\right]_{\beta_{12}=\beta_{13}=0} \equiv } & M_{1}+M_{2}\left(\beta_{14}^{\nu}-1\right), \\
\chi_{2} \equiv \chi\left(\delta_{12}\right)+\left(\beta_{12}^{\nu}-1\right) \chi\left(\delta_{13}\right)+ & \left(\beta_{12}^{\nu}-1\right)\left(\beta_{13}^{\nu}-1\right) \chi\left(\delta_{14}\right) \\
& +M_{2}\left(\beta_{12}^{\nu}-1\right)\left(\beta_{13}^{\nu}-1\right)\left(\beta_{14}^{\nu}-1\right),
\end{aligned}
$$

where $M_{2}$ is free of $\beta_{12}, \beta_{13}, \beta_{14}$. If these $\beta$ 's vanish, $P=0$, so that $\chi_{2}=0$ by definition. Hence (60) gives $M_{2}=0$. Thus

$$
\chi_{2}=\chi(e), \quad e=\delta_{12}+\left(\beta_{12}^{\nu}-1\right) \delta_{13}+\left(\beta_{12}^{\nu}-1\right)\left(\beta_{13}^{\nu}-1\right) \delta_{14} .
$$

The terms independent of the $b$ 's in $\chi_{2}$ are $\chi\left(\beta_{12}^{\nu} \beta_{13} \beta_{14} \beta_{23} \beta_{24} P^{2^{n}-3}\right) \equiv \chi\left\{\left(\beta_{13} \beta_{14} \beta_{23} \beta_{24}+\beta_{12} \beta_{13} \beta_{24} \beta_{34}+\beta_{12} \beta_{14} \beta_{23} \beta_{34}\right) P^{2^{n}-3}\right\}$. For $\beta_{12} \neq 0$, this identity may be written

$$
\chi\left\{t(P-t) P^{2^{n}-3}\right\}=0, \quad t \equiv \beta_{12} \beta_{34},
$$

and is true since the square of $t P^{2^{n}-2}$ equals $t^{2} P^{2^{n}-3}$. For $\beta_{12}=0$, the identity reduces to

$$
\chi\left\{\alpha \beta(\alpha+\beta)^{2^{n}-3}\right\}=0, \quad \alpha \equiv \beta_{13} \beta_{24}, \quad \beta \equiv \beta_{14} \beta_{23} .
$$

In view of (55), it expresses the condition that the quadratic form

$$
x^{2}+(\alpha+\beta) x y+\alpha \beta y^{2} \equiv(x+\alpha y)(x+\beta y)
$$

shall be reducible in the field.

In $e$ the coefficients of $b_{3} b_{4}, b_{4}, b_{4}^{2}$ are the products of $P^{2 n-3}$ by $\beta_{12}^{2}, \beta_{12} \beta_{13} \beta_{23}$, 0 , respectively. Hence, by the symmetry of an invariant, we have*

$$
\chi_{2}=\chi\left\{\left(\sum_{(3)} \beta_{13} \beta_{14} \beta_{23} \beta_{24}+\sum_{(4)} \beta_{12} \beta_{13} \beta_{23} b_{4}+\sum_{(6)} \beta_{12}^{2} b_{3} b_{4}\right) P^{2^{n}-3}\right\}
$$

For a general value of $l$, we shall express $\chi_{l}$ in terms of $\chi_{l-1}$. We develop an auxiliary formula for the case $[1 \ldots 2 l-2] \neq 0, P \equiv[1 \ldots 2 l] \neq 0$. Then, by $\S 15, Q_{2 l}$ can be transformed into (52). Multiplying $x_{2 l-1}$ by $[1 \cdots 2 l-2]$ and $x_{2 l}$ by $P^{-1}$, we get

$$
Q_{2 l-2}+x_{2 l-1} x_{2 l}+\{1 \cdots 2 l-22 l-1\} x_{2 l-1}^{2}+[1 \ldots 2 l-2]^{-2} P^{-2}\{1 \ldots 2 l-22 l\} x_{2 l}^{2} \text {. }
$$

First, let $\chi_{l-1}=0$. Then, by the proof leading to (43), $Q_{2 l-2}$ can be transformed into $\sum_{s=1}^{s=l-1} x_{2 s-1} x_{20}$. Hence $\chi_{l}=\chi(\delta)$, where $\delta$ equals

$$
\delta_{1, \ldots, 2 l-2}=\{1 \ldots 2 l-22 l-1\}\{1 \ldots 2 l-22 l\}[1 \ldots 2 l-2]^{2^{n}-3} P^{2^{n}-3} \text {. }
$$

Next, let $\chi_{l-1}=1$. Then $Q_{2 l-2}$ can be transformed into

$$
\sum_{i=1}^{l-1} x_{2 s-1} x_{2 s}+x_{1}^{2}+d x_{2}^{2}, \quad \chi(d)=1 \text {. }
$$

Hence $\chi_{l}=\chi\left(d+\delta j=1+\chi(\delta)\right.$, where $\delta$ is defined by $\left(63^{\prime}\right)$.

*A merican Journal of Mathematics, loc. cit., p. 267, \& 7, for the special case $P=1$. 
Thus, in either case, $\chi_{l}=\chi_{l-1}+\chi(\delta)$. Hence for any $P$,

$$
\chi_{l}=\chi_{l-1}+\chi(\delta)+\mu\left(P^{\nu}-1\right) \quad \text { when } \quad[1 \cdots 2 l-2] \neq 0 .
$$

Let $P=0$. Then $\chi_{l}=0$ by definition. Thus $\mu=\chi_{l-1}$, and

$$
\chi_{l}=P^{\nu} \chi_{l-1}+\chi\left(\delta_{1, \ldots, 2 l-2}\right) \quad \text { when } \quad[1 \ldots 2 l-2] \neq 0 .
$$

Let $i_{1}, \ldots, i_{2 l}$ give any permutation of $1, \ldots, 2 l$. Then

$$
\begin{gathered}
\chi_{l}=P^{v} \chi_{l-1}+\chi\left(\delta_{i_{1}, \ldots, i_{l l-2}}\right) \quad \text { when } \quad\left[i_{1} \cdots i_{2 l-2}\right] \neq 0, \\
\delta_{i_{1}, \ldots, i_{2 l-2}}=\left\{i_{1} \cdots i_{2 l-2} i_{2 l-1}\right\}\left\{i_{1} \cdots i_{2 l-2} i_{2 l}\right\}\left[i_{1} \cdots i_{2 l-2}\right]^{2^{n}-3} P^{2^{n}-3} .
\end{gathered}
$$

By our usual synthesis, we obtain *

$$
\begin{aligned}
\chi_{l} & =P^{\nu} \chi_{l-1}+\chi\left(d_{1}\right)+\left(P_{1}^{\nu}-1\right) \chi\left(d_{2}\right) \\
& +\left(P_{1}^{v}-1\right)\left(P_{2}^{\nu}-1\right) \chi\left(d_{3}\right)+\cdots+\left(P_{1}^{\nu}-1\right) \cdots\left(P_{\lambda-1}^{\nu}-1\right) \chi\left(d_{\lambda}\right),
\end{aligned}
$$

where $P_{j}$ denotes the pfaffian $\left[i_{1} \ldots i_{2 l-2}\right], d_{j}$ the corresponding function (63), $\lambda$ the number $l(2 l-1)$ of such pfaffians, and $P_{1}, \ldots, P_{\lambda}$ these pfaffians in any sequence.

For $l=3$, set $P_{1}=[1234], P_{2}=[1235], P_{3}=[1236]$. Now

$$
\pi=\left(P_{1}^{v}-1\right)\left(P_{2}^{v}-1\right)\left(P_{3}^{v}-1\right) P
$$

is a factor of the terms of (65) after the fourth. But

$$
P_{1}[1256]-P_{2}[1246]+P_{3}[1245] \equiv \beta_{12} P \text {, }
$$

algebraically. Hence $\pi=0$ if $\beta_{12} \neq 0$. Interchanging 2 and 3 , or 1 and 3 in (66), we find that $\pi=0$ unless $\beta_{12}=\beta_{13}=\beta_{23}=0$. In the latter case, $P_{1}=P_{2}=P_{3}=0$. Then let $P_{4}, \cdots, P_{12}$ denote the pfaffians [ $\left.i j k t\right]$ in which $i, j$ are chosen from $1,2,3$, and $k, t$ from 4, 5,6. Then each $d_{j}$ $(j=4, \ldots, 12)$ has the factor $\{123 k t\}$, which is a linear homogeneous function of $b_{1}, b_{2}, b_{3}$. Among the $P_{j}(j \leqq 12)$ occurs every [1r3s]. Interchanging 2 with $r$ in (66) we conclude, as above, that the terms of (65) with the factor $P \Pi\left(P_{j}^{v}-1\right), j=1, \cdots, 12$, are zero; this is evident if each $\beta_{1 r}=0$, whence $P=0$. Hence $\chi_{3}=P^{v} \chi_{2}+\chi(E)$, where

$$
E=\delta_{1234}+\left(P_{1}^{\nu}-1\right) \delta_{1235}+\left(P_{1}^{\nu}-1\right)\left(P_{2}^{\nu}-1\right) \delta_{1236}+L,
$$

every term of $L$ containing $b_{1}, b_{2}$, or $b_{3}$. In $E$ the coefficients of $b_{5} b_{6}, b_{6}^{2}, b_{6}$ are the products of $P^{2^{n}-3}$ by

$$
[1234]^{2}, \quad 0, \quad V \equiv P_{1}^{v} \psi+\left(P_{1}^{\nu}-1\right) P_{2}^{\nu} \psi,
$$

* The argument initially gives an additional term $\pi M$, where $\pi$ is the product of the $P_{j}^{\nu}-1$ for $j=1, \cdots, \lambda$. But if we set every $P_{j}=0$, we have $P=0$, so that $x_{l}=0$, by definition. Thus $0=M$. 
respectively, where $\psi$ denotes the aggregate $\psi_{123+5}$ of the terms free of the $b$ 's in $\{12345\}$. Evidently $V=\psi$ if either $P_{1} \neq 0$ or $P_{2} \neq 0$. Since $\chi_{3}$ is an absolute invariant, $V$ is symmetrical in $1, \cdots, 5$. Hence $V=\psi$ unless every $\left[i_{1} i_{2} i_{3} i_{4}\right]=0$, where $i, \ldots, i_{4}$ are chosen from $1, \ldots, 5$. But in the latter case, $\psi=0$, by (48), while $V$ obviously vanishes. Hence

$$
\chi_{3}=\chi\left\{\left(F_{3}+\sum \psi_{12345} b_{6}+\sum[1234]^{2} b_{5} b_{6}\right) P^{2^{n}-3}\right\},
$$

where $F_{3}$ may be taken to be

$$
F_{3}=\sum_{(45)} \beta_{13} \beta_{14} \beta_{23} \beta_{24} \beta_{56}^{2}+\sum_{(10)} \beta_{12} \beta_{13} \beta_{23} \beta_{45} \beta_{46} \beta_{56} .
$$

An inspection of the expressions (55), (62), (67) for $\chi_{l}$, for $l=1,2,3$, indicates that, in the formula, given by (65) and (63),

$$
\chi_{l}=\chi\left(C P^{2^{n}-3}\right) \text {, }
$$

$C$ has the following simple relation to the algebraic discriminant $D$ of the quadratic form $Q_{2 l}(\S 10)$. The coefficients of $4 b_{2 l-1} b_{2 l}$ in $4 C$ and $D$ are congruent modulo 2 , likewise the coefficients* of $4 b_{2 l}$; while the terms independent of the $b$ 's in $D-(-1)^{l} P^{2}$ and $4 C$ are multiples of 4 , congruent modulo 8 . In the last statement and in the proofs below, $P$ is the expression obtained from the algebraic pfaffian $[1 \ldots 2 l]$ by giving the various terms any desired signs. For such a function $P$, the congruence

$$
D-(-1)^{2} P^{2} \equiv 4 C \quad(\bmod 8)
$$

uniquely determines $C(\bmod 2)$. The resulting function $(68)$ will be shown to be an absolute invariant of $Q_{2 l}$ in the $G F\left[2^{n}\right]$. Under any linear transformation of determinant $\Delta^{*}, D$ becomes $\Delta^{2} D$ and $P$ becomes $\Delta P+2 \rho(\S 10)$. Hence $d=D-(-1)^{i} P^{2}$ becomes $\Delta^{2} d-(-1)^{l}\left(4 \Delta P \rho+4 \rho^{2}\right)$. But, by (69), $d$ is a multiple of 4 . Hence $d P^{2^{n}-3}$ takes the increment

$$
(\Delta P)^{2^{n-3}}\left(4 \Delta P \rho+4 \rho^{2}\right)+8 S .
$$

Hence in the $G F\left[2^{n}\right]$, the function (68) takes the increment

$$
\chi\left[(\Delta P)^{2^{n}-2} \rho\right]+\chi\left[(\Delta P)^{2^{n-3}} \rho^{2}\right] \equiv 0,
$$

since the quantity in the second brackets is the square of that in the first.

For the canonical form $(41), D=(-1)^{2}(1-4 \delta), P^{2}=1$. Hence

$$
C \equiv \delta, \quad \chi_{\imath} \equiv \chi(\delta)
$$

so that (68) is the desired absolute invariant of $Q_{22}$.

* Such terms, containing a single $b$, cocur when $l>1$. 
The invariants of a quadratic form $Q_{m}$ in the $G F\left[2^{n}\right], \S \S 17-20$.

17. Under the group $G$ of all $m$-ary linear homogeneous transformations in the $G F\left[2^{n}\right]$, we define $C_{0}$ to be the class to which the identically vanishing form $Q_{\mathrm{m}}$ belongs, $C_{2+1}$ the class for (40) with $c=1, C_{2,1}$ the class for (41) with $\chi(\delta)=1, C_{2,0}$ the class for (41) with $\chi(\delta)=0$.

We seek the characteristic absolute invariants $I_{0}, I_{2 l+1}, I_{2,1}, I_{2,0}$ of the general $Q_{m}$ for the respective classes. By (1),

$$
I_{0}=\prod_{i<j}\left(\beta_{i j}^{\nu}-1\right) \Pi\left(b_{i}^{\nu}-1\right) \quad\left(\nu=2^{n}-1\right) .
$$

We shall employ the abbreviations

$$
\pi_{2 l}=\Pi\left(P^{\nu}-1\right), \quad \sigma_{2 l+1}=\Pi\left(S^{\nu}-1\right),
$$

where $P$ ranges over the pfaffians $\left[i_{1} \ldots i_{2 l}\right]$ of order $2 l$, while $S$ ranges over the principal semi-minors $\left\{i_{1} \cdots i_{2 l+1}\right\}$ of order $2 l+1$. In particular,

$$
\begin{gathered}
\pi_{2}=\prod_{i<j}\left(\beta_{i j}^{\nu}-1\right), \quad \sigma_{1}=\Pi\left(b_{i}^{\nu}-1\right), \\
I_{0}=\pi_{2} \sigma_{1} .
\end{gathered}
$$

Since $I_{2 l+1}=1$ if every pfaffian of order $2 l+2$ vanishes, but not every principal semi-minor $\left\{i_{1} \ldots i_{2+1}\right\}$, while $I=0$ in the remaining cases, we have

$$
\begin{gathered}
I_{2 l+1}=\pi_{2++2}\left(1+\sigma_{2+1}\right), \\
\iota_{1}=\pi_{2}+I_{0} .
\end{gathered}
$$

We readily determine the absolute invariant

$$
R_{2 l}=I_{2,1}+I_{21,0},
$$

which has the value 1 or 0 according as $Q_{m}$ is or is not of rank $2 l$. By the result at the end of $\S 12$, we have

$$
R_{2 l}=\pi_{2 l+2} \sigma_{2 l+1}\left(1+\pi_{2 l}\right),
$$

the first two factors being absent if $2 l=m$, so that

$$
R_{\mathrm{m}}=[1 \cdots m]^{v}
$$

( $m$ even ).

By the same reference, we have

$$
I_{2 l, 1}=\pi_{2 l+2} \sigma_{2 l+1} L,
$$

where $L$ is to be determined. Henceforth we consider only sets of coefficients for which every pfaffian of order $2 l+2$ and every $\left\{i \cdots i_{2+1}\right\}$ vanishes; then $I_{2 l, 1}=L$. Consider such a set with $[1 \ldots 2 l] \neq 0$; then $Q_{m}$ can be transformed into the corresponding form $Q_{2 l}$ on $x_{1}, \cdots, x_{2 l}(\S 14)$, and $L$ has the 
value $\chi_{l}$ defined by (68). Let the various pfaffians of order $2 l$ be designated by $P_{1}, \cdots, P_{\imath}$. When $1, \ldots, 2 l$ are replaced by $i_{1}, \ldots, i_{2 l}, P=[1 \ldots 2 l]$ becomes $P_{i}=\left[i_{1} \ldots i_{21}\right]$; let the function $C$ become $C_{i}$. Then for any one of the above sets for which $P_{i} \neq 0, L$ has the value

$$
L_{i}=\chi\left(C_{i} P_{i}^{2^{n}-3}\right) \text {. }
$$

By the usual synthesis of these relations,

$$
L=L_{1}+\left(P_{1}^{v}-1\right) L_{2}+\left(P_{1}^{v}-1\right)\left(P_{2}^{v}-1\right) L_{3}+\cdots+\left(P_{1}^{v}-1\right) \cdots\left(P_{t-1}^{v}-1\right) L_{t},
$$

with initially an addition term $M \Pi\left(P_{i}^{\nu}-1\right), i=1, \ldots, t$. But for every $P_{i}=0, I_{2 l, 1}$ is zero, by definition, so that $M=0$. Similarly,

$$
I_{2 l, 0}=\pi_{2 l+2} \sigma_{2 l+1} K \text {. }
$$

For the sets with every $\left[i_{1} \cdots i_{2 l+2}\right]=\left\{i_{1} \cdots i_{2 l+1}\right\}=0$, we have $I_{2 l, 0}=K$. If also $P_{i} \neq 0$, then $\dot{K}=1+L_{i}$. A synthesis of the latter relations gives

$$
K=1+L+\mu \prod_{i=1}^{t}\left(P_{i}^{v}-1\right) .
$$

If every $P_{i}=0$, then $I_{2 l, 0}=0$, by definition. Hence $\mu=1$. Thus

$$
I_{2 l, 0}=\pi_{2 l+2} \sigma_{2 l+1}\left(L+1+\pi_{2 l}\right) .
$$

As a check, we note that (74) follows at once from (75) and (78); also that for $m=3, I_{2,1}$ is the invariant $F$ given for $n \leqq 4$ in the American Journal of Mathematics, l. c.

18. The preceding determination of the invariant $I_{2 l, 1}$ was based upon the second method (§14) of reducing quadratic forms in the $G F\left[2^{n}\right]$. For $l>1$, a determination based upon the first method $(\$ 12)$ appears to be more complicated. We shall treat briefly the case $l=1, m=4$, from the latter standpoint; we are thereby led naturally to a noteworthy modification of the earlier formula for $I_{2,1}$. If $\beta_{12} \neq 0, Q_{4}$ is transformable into an irreducible binary form $B=x_{1} x_{2}+x_{1}^{2}+c x_{2}^{2}$ if and only if $P \equiv[1234]=0,\{123\}=0,\{124\}=0$, and (55) is unity. In general, if $\beta_{i j} \neq 0, I_{2,1}=1$ if and only if $P=0$, $\{i j k\}=0,\{i j t\}=0, L_{i j}=1$, where $i, j, k, t$ form a permutation of $1,2,3,4$, and

$$
L_{i j}=\chi\left(b_{i} b_{j} \beta_{i j}^{2^{n}-3}\right), \quad G_{i j}=\left(\{i j k\}^{\nu}-1\right)\left(\{i j t\}^{\nu}-1\right) L_{i j} .
$$

Hence, if $\beta_{i j} \neq 0, I_{2,1}=\left(P^{v}-1\right) G_{i j}$. By the usual synthesis,

$$
\begin{aligned}
I_{2,1}=\left(P^{\nu}-1\right)\left\{G_{12}+\left(\beta_{12}^{\nu}-1\right) G_{13}\right. & +\left(\beta_{12}^{\nu}-1\right)\left(\beta_{13}^{\nu}-1\right) G_{14} \\
& \left.+\cdots+\left(\beta_{12}^{\nu}-1\right) \cdots\left(\beta_{24}^{\nu}-1\right) G_{34}\right\} .
\end{aligned}
$$


A comparison of (80) with (75) and (77) indicates that

$$
\left(P^{\nu}-1\right) G_{i j}=\left(P^{\nu}-1\right) \sigma_{3} L_{i j}, \quad \sigma_{3} \equiv \prod_{(4)}\left(\{i j k\}^{\nu}-1\right) .
$$

This equality is obvious except in the case $P=0,\{i j k\}=0,\{i j t\}=0, L_{i j}=1$. But then $Q_{4}$ is transformable into $B$, so that $\{i k t\}=0,\{j k t\}=0$.

19. To determine the relative invariants of $Q_{m}$ in the $G F\left[2^{n}\right]$, we note that (end of $\S 12$ ) the only classes under the total group $G$ which separate into subclasses under the group $G_{1}$ of determinant unity are $C_{m, 1}$ and $C_{m, 0}$ for $m$ even, while the only such class is $C_{m}$ for $m$ odd. In the latter case, the sub-classes may be designated by $K_{m, c}$, a representative form being (40) for $m=2 l+1$. Here $c$ is the non-vanishing semi-discriminant of $Q_{m}$. As in $\S 9$, there exists a single invariant of a given weight $w, 0<w<\nu$, since it must vanish for all the classes other than the $K_{m, c}$, and since its value for $K_{m, c}$ must be $c^{k / 2}$ times its value $\neq 0$ for $K_{m, 1}$. But such an invariant is $S^{w / 2}$ for $w$ even and $S^{(w+v) / 2}$ for $w$ odd, where $S$ is the semi-discriminant of the general form $Q_{m}$. Hence for $m$ odd, the only relative invariants are powers of the semi-discriminant.

Next let $m$ be even. The sub-classes mentioned above may be designated $K_{m, \chi}$, , where $\chi=1$ or 0 , and $P=[1 \cdots m] \neq 0$, representative forms being

$$
\sum_{\delta=2}^{m} x_{2 s-1} x_{2 s}+P x_{1} x_{2}+P^{2} x_{1}^{2}+\delta x_{2}^{2} \quad[x(\delta)=\chi] .
$$

To obtain an invariant $\phi$ of weight $w, 0<w<\nu$, we must assign to $\phi$ the value 0 for all the classes other than the $K_{m, x, P}$, and for the latter $P^{w}$ times the value of $\phi$ for $K_{m, x, 1}$. Hence the two values for the last two classes $(x=1$ or 0$)$ alone are arbitrary, so that there are just two linearly independent invariants of the given weight $w$. But $P^{w}$ and $P^{w} \chi_{m / 2}$ have these properties, $\chi_{m / 2}=I_{m, 1}$ being the absolute invariant determined in $\$ 16$. For $m$ even, the only relative invariants are

$$
[1 \cdots m]^{\infty}\left(c_{1}+c_{2} \chi_{m / 2}\right) \quad\left(0<w<2^{n}-1, c_{1} \text { and } c_{2} \text { constants }\right) .
$$

20. A simple method of obtaining a smaller number of independent invariants of $Q_{m}$ in the $G F\left[2^{n}\right]$, in terms of which all the above invariants can be expressed rationally, will be illustrated by the case $m=4$. Let

$$
F_{4}=I_{4,1}+I_{3}, \quad J_{4}=I_{2,1}+I_{0}, \quad P=[1234] .
$$

By (72) for $l=1$, and (75) for $l=2$,

$$
I_{3}=\left(P^{\nu}-1\right)\left(1+\sigma_{3}\right), \quad I_{4,1}=L \equiv \chi_{2}, \quad \sigma_{3} \equiv \prod_{(1)}\left(S^{\nu}-1\right) .
$$


Since $\chi_{2}$, given by (62), is a multiple of $P,\left(P^{v}-1\right) I_{4,1}=0$. Hence*

$$
\chi_{2}=I_{4,1}=P^{v} F_{4,} \quad I_{3}=\left(P^{v}-1\right) F_{4}, \quad I_{4,0}=P^{v}\left(F_{4}+1\right),
$$

the last following from $I_{4,1}+I_{4,0}=P^{\nu}$, given by (73) and $\left(74^{\prime}\right)$ for $l=2$. By (73) and (74) for $l=1$,

$$
I_{2,1}+I_{2,0}=\left(P^{v}-1\right) \sigma_{3}\left(1+\pi_{2}\right)=\left(P^{v}-1\right) \sigma_{3}+\pi_{2},
$$

since each $\pi_{2} S=0, \pi_{2} P=0$, in view of the form $\left(71_{1}^{\prime}\right)$ of $\pi_{2}$. But by $\left(82_{1}\right)$ and $\left(83_{2}\right)$,

$$
\left(P^{v}-1\right) \sigma_{3}=I_{3}+P^{v}-1=\left(P^{v}-1\right)\left(F_{4}+1\right) \text {. }
$$

Hence we have

$$
I_{2,1}+I_{2,0}=\left(P^{v}-1\right)\left(F_{1}+1\right)+\pi_{2} .
$$

Now each term of (80) has a factor $\beta_{i j}$, in view of the $L_{i j}$. Thus

$$
\pi_{2} I_{2,1}=0, \quad \pi_{2} I_{0}=I_{0},
$$

the latter following from $\left(70^{\prime}\right)$. Hence by $\left(81_{2}\right)$

$$
I_{0}=\pi_{2} J_{4}, \quad I_{2,1}=J_{4}\left(\pi_{2}+1\right) .
$$

Then by (72') and (84),

$$
I_{1}=\pi_{2}\left(J_{1}+1\right), \quad I_{8,0}=\left(P^{v}-1\right)\left(F_{4}+1\right)+J_{4}\left(\pi_{2}+1\right)+\pi_{2} .
$$

Hence formulæ (83), (85), (86) express all the absolute invariants of $Q_{4}$ in terms of $F_{4}, J_{4}, P, \pi_{2}$. Incorporating the result at the end of $\S 19$, we have the

TheOREM. $t$ Every invariant of $Q_{4}$ in the $G F\left[2^{n}\right]$ is an integral function of the invariants $F_{4}, J_{4}, P, \pi_{2}$; in fact, a linear homogeneous function of

$$
J_{4}, \pi_{2}, \pi_{2} J_{4}, P^{\infty}, P^{0} F_{4} \quad\left(e=0,1, \cdots, 2^{n}-1\right) \text {. }
$$

The values-of these invariants for the various classes are here shown.

\begin{tabular}{c|c|c|c|c|c|c|c} 
& $K_{4,0, P}$ & $K_{4,1, P}$ & $C_{3}$ & $C_{2,0}$ & $C_{2,1}$ & $C_{1}$ & $C_{0}$ \\
\hline$F_{4}$ & 0 & 1 & 1 & 0 & 0 & 0 & 0 \\
$J_{1}$ & 0 & 0 & 0 & 0 & 1 & 0 & 1 \\
$P$ & $P$ & $P$ & 0 & 0 & 0 & 0 & 0 \\
$\pi_{2}$ & 0 & 0 & 0 & 0 & 0 & 1 & 1
\end{tabular}

* Another prool of $\left(83_{1}\right)$ results from the laot that $P v F_{4}$ has the value 1 for olass $C_{4,1}$ and the value 0 for the remaining olaeses.

† Another proof follow from the fact that the second member of (84) has the value 1 for claseses $C_{2,1}$ and $C_{2,0}$, the value 0 for the remaining clasece.

$\ddagger$ For $n=1$, Proceedings of the London Mathomatioal Sooiety, vol. 5 (1907), pp. 303-311. The invariants $A_{4}, I_{4}$ are the $\pi_{2}, I_{0}$ of the present paper. The explioit expreesions for $J_{4}$ and $F_{4}$ are given on p. 308 and p. 310. 
To prove (end of $\S 5$ ) the independence of $F_{4}$, we employ $C_{3}$ and $C_{2,0}$; for $J_{1}, C_{1}$ and $C_{0}$; for $P, K_{4,1, P}$ and $C_{3}$; for $\pi_{2}, C_{2,0}$ and $C_{1}$. Hence the four invariants are independent. It follows from the table that

$$
F_{4} J_{4}=F_{4} \pi_{2}=P J_{4}=P \pi_{2}=0, \quad P^{2^{n}}=P, \quad I^{2}=I\left(I=F_{4}, J_{4} \text { or } \pi_{2}\right) .
$$

Any product of two invariants (87) reduces to one of the set by means of (88).

Reduction of binary cubic forms in the $G F\left[p^{n}\right]$.

21. If $p^{n}=3 l+2$ or $3^{n}$, every element $\neq 0$ of the $G F\left[p^{n}\right]$ is a cube:

$$
e=e^{-s t} \quad \text { or } \quad e^{3.3 n-1},
$$

respectively; hence every element has an unique cube root in the field. But if $p^{n}=3 l+1$, just one-third of the elements $\neq 0$ are cubes. If $\epsilon$ is a primitive root in the field, the cubes are $e^{3 i}(i=1, \ldots, l)$; while the not-cubes are the products of the preceding by $\epsilon, \epsilon^{2}$. We shall set

$$
\beta=1 \text { if } p^{n}=3 l+2 \text { or } p^{n}=3^{n} ; \quad \beta=1, \epsilon \text { or } \epsilon^{2} \text { if } p^{n}=3 l+1 .
$$

Consider the binary cubic with coefficients in the $G F\left[p^{n}\right]$,

$$
f(x, y) \equiv a_{0} x^{3}+a_{1} x^{2} y+a_{2} x y^{2}+a_{3} y^{3} .
$$

If $\rho_{1}, \rho_{2}, \rho_{3}$ are the roots of $f(x, 1)=0$, the discriminant of $(90)$ is

$$
D=a_{0}^{4} \Pi\left(\rho_{i}-\rho_{j}\right)^{2}=18 a_{0} a_{1} a_{2} a_{3}-4 a_{0} a_{2}^{3}-4 a_{1}^{3} a_{3}+a_{1}^{2} a_{2}^{2}-27 a_{0}^{2} a_{3}^{2} \text {. }
$$

When $p^{n}=2$ the special form $f_{1}=x y(x+y)$ is unaltered under every linear transformation in the field; it is a special case of the canonical form (93). Except when $f \equiv 0$, or when $p^{n}=2$ and $f=f_{1}$, we can transform $f$ into a form $f^{\prime}$ with $a_{0}^{\prime} \neq 0$. If $a_{0}=0, a_{3} \neq 0$, we apply $(y,-x)$. If $a_{0}=a_{3}=0$, we apply $(x, \lambda x+y)$ and have $a_{0}^{\prime}=a_{1} \lambda+a_{2} \lambda^{2}$.

In the normalization of a form $f$ with $a_{0} \neq 0$, we consider several cases.

(i) In case there is a triple root $\rho$, we apply the transformation

$$
x-\rho y=\alpha x^{\prime}, \quad y=\alpha^{-1} y^{\prime},
$$

of determinant unity. We obtain $a_{0} \alpha^{3} x^{\prime 3}$, and hence $\beta x^{3}$.

(ii) In case there is a double root $\rho_{1}$ and a simple root $\rho_{2}$, we apply the transformation of determinant unity

$$
x-\rho_{1} y=\alpha x^{\prime}, \quad x-\rho_{2} y=\alpha^{-1}\left(\rho_{1}-\rho_{2}\right) y^{\prime} .
$$

We obtain $c x^{\prime 2} y^{\prime}$, where $c=a_{0} \alpha\left(\rho_{1}-\rho_{2}\right)$ may be made unity by choice of $\alpha$.

(iii) Let $f(x, 1)=0$ have three distinct roots $\rho_{i}$ in the field. Applying the 
transformation (92) to $f(x, y)$, we obtain $k x^{\prime} y^{\prime}\left(x^{\prime}+r y^{\prime}\right)$, where

$$
k=a_{0} \alpha\left(\rho_{3}-\rho_{2}\right), \quad r=\alpha^{-2}\left(\rho_{1}-\rho_{2}\right)\left(\rho_{3}-\rho_{1}\right) /\left(\rho_{2}-\rho_{3}\right) .
$$

We make $k=1$ by choice of $x$. Then $r^{2}=D$, by (91). In

$$
x y(x+r y), \quad r^{2}=D,
$$

we may change the sign of $r$ by applying $\left(r y,-r^{-1} x\right)$. Under the group $G_{1}$ of binary transformations of determinant unity, the canonical form is (93), where $r$ is a particular square root of $D$.

Under the total group $G$, a canonical form is

$$
\beta x y(x+y) \quad \text { or } \quad x y(x+\beta y) .
$$

Indeed, $\left(s x, r^{-1} s y\right)$ replaces (93) by $c x y(x+y), c=r^{-1} s^{3}$.

(iv) Let $f$ be the product of an irreducible quadratic factor and a linear factor. The latter may be taken to be a multiple of $x$.

If $p \neq 2$, we apply $(x, y+a x)$ and obtain

$$
d x\left(y^{2}-\mu x^{2}\right), \quad \mu \text { a not-square. }
$$

Applying $\left(d x, d^{-1} y\right)$, we obtain the canonical form under $G_{1}$

$$
x\left(y^{2}-\sigma x^{2}\right), \quad \sigma \text { a not-square, } \sigma=1 D .
$$

As the canonical form under the total group $G$, we may take *

$$
x\left(y^{2}-\nu \beta^{2} x^{2}\right), \quad \nu \text { a particular not-square. }
$$

The latter is obtained from (94) with $\sigma=\nu t^{2}$ by applying $\left(s^{-2} x, s y\right)$, where $s$ is chosen to make $t s^{-3}$ take one of the values $\beta$.

If $p=2, f=d x\left(x y+a x^{2}+b y^{2}\right), d a b \neq 0$. Applying $\left(b^{b} x, b^{-k} y\right)$, we obtain a form $m x\left(x y+y^{2}+\delta x^{2}\right)$. Applying $(x, y+t x)$, we find that $\delta$ is replaced by $c=\delta+t+t^{2}$. This equation is solvable for $t$ in the $G F\left[2^{n}\right]$ if and only if $\chi(c)=\chi(\delta)$, where

$$
\chi(c)=\sum_{i=0}^{n-1} c^{2}, \quad \chi^{2} \equiv \chi, \quad \chi \equiv 0 \text { or } 1 \text {. }
$$

If $\chi(\delta)=0$, the quadratic factor would be reducible. Hence

$$
m x\left(x y+y^{2}+c x^{2}\right), \quad c \text { a particular root of } \chi(c)=1,
$$

are the canonical forms under $G_{1}$. If we multiply $x$ and $y$ by a suitably chosen element, we obtain as the canonical form under the total group $G$

$$
\beta x\left(x y+y^{2}+c x^{2}\right) \text {. }
$$

* We may take $\beta x\left(x^{2}-\nu y^{2}\right)$, obtained from (94) with $\sigma=\nu t^{2}$ by applying ( $\left.a x, v a t y\right)$, where $a$ is chosen to make $-a^{3} v t^{2}=\beta$. 
(v) Finally, let $f$ be irreducible in the $G F\left[p^{n}\right]$. Then $f(x, 1)=0$ has a root $\rho$ in the $G F\left[p^{3 n}\right]$, the remaining roots being $\rho^{p^{n}}, \rho^{p^{2 n}}$. For

$$
x=\alpha x^{\prime}+\beta y^{\prime}, \quad y=\gamma x^{\prime}+\delta y^{\prime} \quad(\Delta=\alpha \delta-\beta \gamma+0),
$$

we have

$$
x-\rho y=(\alpha-\rho \gamma)\left(x^{\prime}-\lambda y^{\prime}\right), \quad \lambda \equiv \frac{\delta \rho-\beta}{-\gamma \rho+\alpha}
$$

so that $\rho$ is transformed by the inverse of the linear fractional form of (97). Hence when $\alpha, \beta, \gamma, \delta$ take all values in the $G F\left[p^{n}\right]$ for which $\Delta \neq 0$, the fraction $\lambda$ takes $p^{n}\left(p^{2 n}-1\right)$ distinct values in the $G F\left[p^{3 n}\right]$, no one belonging to the $G F\left[p^{n}\right]$. Hence $\lambda$ can be made equal to any assigned element of the $G F\left[p^{3 n}\right]$, not occurring in the $G F\left[p^{n}\right]$; the ratios of the cotficients in (97) are thereby uniquely determined. The canonical forms under the total group $G$ of all transformations (97) are therefore $\beta C$, where $C$ is a particular irreducible cubic $x^{3}+\cdots$. We may take

$$
C=x^{3}-x y^{2}+\tau y^{3},
$$

where $\tau$ is suitably chosen. For, if $z^{3}-z+\tau=0$ were reducible for every $\tau$ in the $G F\left[p^{n}\right], z^{3}-z$ would take $p^{n}$ distinct values when $z$ does, whereas $z^{3}-z$ vanishes for $z=0, \pm 1$.

It remains to discuss the normalization of $f$ under the group $G_{1}$ of the $\left(p^{2 n}-1\right) p^{n}$ transformations (97) with $\Delta=1$. Then, in (98), $\lambda=\rho$ only when

$$
\alpha=\delta= \pm 1, \quad \beta=\gamma=0 \text {. }
$$

For $p=2$, the corresponding transformation (97) is the identity, and $\lambda$ takes $p^{n}\left(p^{2 n}-1\right)$ distinct values; the $2^{n}-1$ canonical forms are therefore $m C$. Henceforth, let $p>2$. Then $\lambda$ takes only $\frac{1}{2} p^{n}\left(p^{2 n}-1\right)$ values. Hence the roots of the various irreducible cubic equations fall into two types. Since $\Delta=1$, $\lambda=-\delta \gamma^{-1}+\gamma^{-1}(\alpha-\gamma \rho)^{-1}, \quad \lambda-\lambda p^{n}=\left(\rho-\rho^{\prime^{n}}\right)(\alpha-\gamma \rho)^{-1}\left(\alpha-\gamma \rho^{p^{n}}\right)^{-1}$, when $\gamma \neq 0$. But the second equation is true also when $\gamma=0$. Thus

$$
\phi(\lambda)=\phi(\rho) \div a_{0}^{-2} f^{2}(\alpha, \gamma), \quad \phi(\rho) \equiv\left(\rho-\rho^{p^{n}}\right)\left(\rho^{p^{n}}-\rho^{p^{2 n}}\right)\left(\rho^{p^{2 n}}-\rho\right) .
$$

Since $\phi^{p^{n}}=\phi, \phi(\rho)$ is an element of the $G F\left[p^{n}\right]$; in particular, it is unaltered when $\rho$ is replaced by another root $\rho^{p \hat{n}}$ or $\rho^{p^{2 n}}$ of the same cubic. Hence if $\rho$ and $\lambda$ are roots of two irreducible cubic equations such that $\lambda$ is a linear fractional function of $\rho$ of determinant a square (which may evidently be made unity), the ratio of $\phi(\lambda)$ to $\phi(\rho)$ is a non-vanishing square in the field. But if this determinant is a not-square, the ratio of the $\phi$ 's is a not-square. It suffices to prove the latter for a particular linear fractional transformation of determinant a not-square $\nu$, for example, $\lambda=\nu \rho$, whence $\phi(\lambda)=\nu^{3} \phi(\rho)$. Further, it was 
shown above that there exists a linear fractional transformation replacing $\rho$ by any root of any irreducible cubic. Hence if $\rho$ and $\lambda$ are roots of the equations. corresponding to two irreducible cubic forms $C$ and $C^{\prime}$, then $C$ is equivalent to a multiple of $C^{\prime}$ under the group $G_{1}$ if and only if the ratio of $\phi(\lambda)$ to $\phi(\rho)$ is a square.

Since there are $\frac{1}{6}\left(p^{2 n}-1\right) p^{n}$ distinct cubic equations of each type, there are exactly 6 binary transformations of determinant unity which multiply a given $C$ by a constant, necessarily \pm 1 by $\left(99_{1}\right)$.

Under $G_{1}$ the non-equivalent canonical forms may be taken to be $m C_{1}$ and $m C_{2}$, where $C_{1}$ and $C_{2}$ are particular irreducible cubic forms $x^{3}+\cdots$, such that the equation corresponding to $C_{1}$ has a root $\rho$ with $\phi(\rho)=1$, that corresponding to $C_{2}$ a root $\rho$ with $\phi(\rho)=\nu$, where $\nu$ is a fixed not-square. Further, $m$ takes only one of each pair of non-vanishing values $\pm M$. A cubic form, for which the invariant $E(\S 22)$ is not zero, can be transformed into $m C_{1}$ or $m C_{2}$ according as $E=m^{2}$ or $m^{2} \nu$.

The invariants of the binary cubic form in the $G F\left[p^{n}\right], \S 22-26$.

22. The results under case (v) of $\S 21$ lead us quite naturally to an important invariant $E$ of the binary cubic $f(x, y)$ under the group $G_{1}$ of transformations of determinant unity. According to our general standpoint, there exists an invariant which takes prescribed values for each class under $G_{1}$. Let $E$ be zero for all reducible cubics, $E=m^{2}$ for the cubic $m C_{1}, E=m^{2} \nu$ for $m C_{2}$. Thus $E$ has the value $E_{i}=m^{2} \phi(\rho)$ for $m C_{i}(i=1$ or 2$)$. We readily deduce the value of $E$ for any irreducible cubic $f(x, y)$. One of the cubics $m C_{i}$ can be transformed with $G_{1}$ into $f$. If $\lambda$ is a root of $f(x, 1)=0,\left(99_{1}\right)$ gives $\phi(\lambda)=\phi(\rho) m^{2} / a_{0}^{2}$, since the coefficient $a_{0}$ of $f(x, y)$ is the value of $m C_{i}$ for $x=\alpha, y=\gamma$. Hence $a_{0}^{2} \phi(\lambda)=E_{i}$. Thus, for any irreducible cubic $f(x, y)$, $E$ has the value $a_{0}^{2} \phi(\lambda)$. For a reducible cubic, $E=0$ by definition. Hence * for an arbitrary cubic $f(x, y), E=a_{0}^{2} R$, where $R$ is the resultant of $x^{p n}=x$ and $f(x, 1)=0$, the constant factor being determined so that

$$
R=\prod_{i=1}^{3}\left(x_{i}-x_{i}^{p p}\right),
$$

where the $x_{i}$ are the roots $f(x, 1)=0$. Under the transformation $(x, \delta y)$, each root is multiplied by the determinant $\delta$; then $R$ is multiplied by $\delta^{3}$, while $a_{0}$ is unaltered. Hence $E$ is a relative invariant of weight 3 .

23. We seek absolute characteristic invariants of the $d$ classes represented by $\beta x^{3}$, where $d$ is the greatest common divisor of $\mu=p^{n}-1$ and 3 , and $\beta$ is de-

\footnotetext{
* For a different, but equivalent, definition of $E$, see these Transactions, loc. oit., p. 307. For the explicit expression for $E$ for various values of $p^{n}$, see pp. 208, 212, 229, 230, 231.
} 
fined by (89). To this end we construct a polynomial $Q$ in the $a_{i}$ which has the value $\beta^{\mu / d}$ for the class $\beta x^{3}$ (for each of the $d$ values of $\beta$ ) and the value zero for all classes other than these $d$; then $Q$ will be an absolute invariant of the cubic form. If $d=1, Q$ itself is the desired characteristic invariant. If $d=3, \omega=\epsilon^{\mu / 3}$ is a cube root of unity in the field, and

$$
\frac{1}{3}\left(Q+Q^{2}+Q^{3}\right), \quad \frac{1}{3}\left(\omega^{2} Q+\omega Q^{2}+Q^{3}\right), \quad \frac{1}{3}\left(\omega Q+\omega^{2} Q^{2}+Q^{3}\right)
$$

are characteristic absolute invariants for the classes $x^{3}, e x^{3}, \epsilon^{2} x^{3}$, respectively.

The cubic form $f(x, y)$, given by (90), has a triple root if and only if

$$
A=a_{1}^{2}-3 a_{0} a_{2}, \quad B=a_{2}^{2}-3 a_{1} a_{3}, \quad C=a_{1} a_{2}-9 a_{0} a_{3}
$$

all vanish in the field, a result valid for any $p$. Let

$$
\pi=\left(1-A^{\mu}\right)\left(1-B^{\mu}\right)\left(1-C^{\mu}\right) .
$$

Evidently $Q \equiv \pi q$. To determine $q$, we consider the sets $a_{i}$ for which $A, B$, $C$ all vanish, so that $Q=q$. For the sets with $a_{0} \neq 0, f$ can be transformed into $a_{0} x^{3}$, so that $Q=a_{0}^{\mu / d}$. Hence

$$
Q \equiv a_{0}^{\mu / d}+c\left(1-a_{0}^{\mu}\right),
$$

for all the sets. For the sets with $a_{0}=0, a_{3} \neq 0, f \equiv a_{3} y^{3}$, so that

$$
Q=a_{3}^{\mu / d}=c, \quad c \equiv a_{3}^{\mu / d}+k\left(1-a_{3}^{\mu}\right) .
$$

Then for the sets with $a_{0}=a_{3}=0, f \equiv 0, Q=0$, whence $k=0$. Thus

$$
Q=\pi\left[a_{0}^{\mu / d}+a_{3}^{\mu / d}\left(1-a_{0}^{\mu}\right)\right] \text {. }
$$

For $d=1$, the second factor of $Q$ equals $1-t$, where

$$
t=\left(a_{0}^{\mu}-1\right)\left(a_{3}^{\mu}-1\right) \text {. }
$$

Since $a\left(a^{\mu}-1\right)=0$, we have $\pi t=I_{0}$,

$$
\begin{gathered}
I_{0}=\prod_{i=0}^{8}\left(a_{i}^{\mu}-1\right), \quad P=\left(a_{1}^{\mu}-1\right)\left(a_{2}^{\mu}-1\right), \\
Q=\pi-I_{0}
\end{gathered}
$$

In particular, if $p=3$ (whence $d=1$ ), $\pi=P$.

For $d=3$, the cube of the final factor in (104) is $1-t$. Hence

$$
Q^{3} \equiv \pi-I_{0}, \quad Q^{4}=Q
$$

(for any $d)$.

24. Let $J$ be the characteristic absolute invariant of the class represented by $x^{2} y$. Then $J=\left(1-D^{\mu}\right) L$. Consider the sets $a_{i}$ with $D=0, A \neq 0$, and then the sets with $D=A=0, B \neq 0$. We find that

$$
L=1+m\left(A^{\mu}-1\right)\left(B^{\mu}-1\right) \text {. }
$$


Consider the sets with $D=A=B=0$. Then $C=0$, since

$$
-3 D \equiv C^{2}-4 A B \text {, }
$$

so that $0=1+m$. Hence

$$
J=\left(1-D^{\mu}\right)\left\{1-\left(1-A^{\mu}\right)\left(1-B^{\mu}\right)\right\} .
$$

We may give $J$ a more symmetrical form. If $p \neq 3$,

$$
D^{\mu}=\left(C^{2}-4 A B\right)^{\mu},
$$

by (108). Also $A\left(A^{\mu}-1\right)=0$. Hence, by (103),

$$
J=1-D^{\mu}-\pi \text {. }
$$

This result is true also if $p=3$, since then $D P=0, \pi=P$.

In view of (110), $\pi$ is an absolute invariant of the cubic.

25. We can now prove that every invariant of the binary form in the $G F\left[p^{n}\right], p>2$, is a rational integral function of $D, E, Q, I_{0}$. Under the group $G_{1}$ of transformations of determinant unity, a complete set of nonequivalent classes are defined by the representative forms $f \equiv 0, \beta x^{3}, x^{2} y$, (93), (94), $m C_{1}, m C_{2}$, in which $r$ and $m$ each take $\frac{1}{2} \mu$ values $\neq 0$, no one value being the negative of another. Here $\mu=p^{n}-1$. Hence, for $p>2$, the number of classes is

$$
1+d+1+\frac{1}{2} \mu+\frac{1}{2} \mu+\frac{1}{2} \mu+\frac{1}{2} \mu=d+2+2 \mu .
$$

Hence by $\S 4$ there are exactly $d+2+2 \mu$ linearly independent invariants under $G_{1}$. These may be taken to be

$$
I_{0} Q^{\delta}(\delta=1, \cdots, d), J, D^{i}, E^{i}(i=1, \ldots, \mu) .
$$

We note that $I_{0}$ and $J$ are characteristic invariants of the classes $f \equiv 0, x^{2} y$; likewise $Q$ for $x^{3}$ if $d=1$, and the linear combinations (101) for the $\beta x^{3}$ if $d=3$. Suitable linear combinations of the $E^{i}$ give the characteristic invariants of the classes $m C_{f}$, while linear combinations of the $\left(E^{\mu}-1\right) D^{i}$ give those for the classes (93) and (94). For an irreducible cubic, $D=E^{2}$ by (91) and (100). Hence for every cubic,

$$
D E=E^{3}, \quad D^{i} E^{k}=E^{k+2 i}, \quad\left(E^{\mu}-1\right) D^{i}=E^{2 i}-D^{i} .
$$

Hence, as in $\S 4$, the invariants (111) are linearly independent.* Of these,

* If $N$ is the number of sets of solutions $x: y$ of $f(x, y)=0$ in the $G F\left[p^{n}\right]$, there exists an absolnte invariant $K$ for which $K=N-1(\bmod p)$, with $K=0$ if $f$ is identically zero, Bulletin of the American Mathematioal Society, vol. 14 (1908), p. 316. For $p>2$, we may give to $K$ the comprot expression

$$
K=J-E^{\mu}+\left(1-E^{\mu}\right)\left(D^{\mu}+D^{\mu \mu}\right) .
$$


$I_{0}, Q$ and $J$ are absolute invariants, while the powers of $D$ and $E$ are relative invariants under the total group $G$.

TheOREM. The $d+2 p^{n}$ invariants (111) give a complete set of linearly independent invariants of the binary cubic form in the $G F\left[p^{n}\right], p>2$.

We may suppress $J$ and introduce 1 , in view of (107), (110). We may suppress $Q^{d}$ and introduce $\pi$, etc. Any product of $D, E, \pi, Q, I_{0}$ can be reduced to a linear homogeneous function of the invariants (111), by means of the relations (valid for any $p$ ):

$$
\begin{aligned}
& D E=E^{3}, \quad D \pi=D Q=D I_{0}=E \pi=E Q=E I_{0}=Q I_{0}=0, \\
& \pi Q=Q, \quad \pi I_{0}=I_{0}, \quad I_{0}^{2}=I_{0}, \quad Q^{4}=Q, \quad D^{\mu+1}=D, \quad E^{\mu+1}=E .
\end{aligned}
$$

Additional invariants of a cubic in the $G F\left[2^{n}\right]$.

26. For $p=2$, there are $d+2+3 \mu$ classes represented by $f \equiv 0, \beta x^{3}$, $x^{2} y,(93),(96)$, and in $m C$, with $\mu=2^{n}-1$ forms in each of the last three sets. Hence we require $\mu$ invariants in addition to (111). These additional invariants together with the powers of $D$ should enable us to differentiate the $2 \mu$ classes represented by (93) and (96), and hence to distinguish between the types of reducible cubics having no double root.

We seek the necessary and sufficient conditions that $f(x, y)$ shall be the product of a linear and an irreducible quadratic factor in the $G F\left[2^{n}\right]$. If $a_{0}=0, a_{1} x^{2}+a_{2} x y+a_{3} y^{2}$ must be irreducible, so that, by (42), (55),

$$
\chi\left(a_{1} a_{3} a_{2}^{2 n-3}\right)=1 \text {. }
$$

Next, let $a_{0} \neq 0$. Let $a_{0} x+a_{1} y=\xi, y=\eta$. Then

$$
a_{0}^{2} f(x, y)=\xi+e \xi \eta^{2}+g \eta^{3}, \quad e=a_{1}^{2}+a_{0} a_{2}, \quad g=a_{0} D^{\sharp} .
$$

If $e=0$, the conditions require that there exist one and but one cube root of $g$; hence there must be a single root of $\omega^{3}=1$, so that $2^{n}-1$ must be prime to 3 . When the latter condition is satisfied, every element is a cube ( $\$ 21)$. Hence if $e=0$, the desired necessary and sufficient conditions are that $n$ be odd and that $g \neq 0$. Finally, let $e \neq 0$. Let $X=e^{-t} \xi, Y=\eta$. Then

$$
e^{-1} a_{0}^{2} f(x, y)=X^{3}+X Y^{2}+t Y^{3}
$$$$
\left(t=g e^{-i}\right) .
$$

If $t=0$, we obtain $X(X+Y)^{2}$. Hence must $t \neq 0$. Our problem thus reduces to the characterization of the values $\neq 0$ of $t$ for which there is one and but one root in the $G F\left[2^{n}\right]$ of the equation

$$
z^{3}=z+t \text {. }
$$

We shall investigate the relations between the $\alpha$ 's and $\beta^{\prime} s$ in

$$
z^{2^{k}}-z=\alpha_{k}^{2} z^{2}+\beta_{k} z \text {. }
$$

Trans. Am. Math. Soc. 11 
We have the initial sets of values

$$
\alpha_{1}=\beta_{1}=1, \quad \alpha_{2}=1, \quad \beta_{2}=t+1, \quad \alpha_{3}=\beta_{3}=t+1 .
$$

Squaring (117) and eliminating $z^{4}$, we find that

$$
\alpha_{k+1}=\alpha_{k}^{2}+\beta_{k}+1, \quad \beta_{k+1}=t \alpha_{k}^{4}+1 .
$$

As $k$ increases, the expressions for the $\alpha_{k}, \beta_{k}$ in terms of $t$ increase rapidly in complexity. We shall prove by induction that

$$
\beta_{k}=\alpha_{k}^{2}+t \alpha_{k} \alpha_{k-1}^{2} \text {. }
$$

The expression for $\beta_{k+1}$ analogous to (120) will equal $\left(119_{2}\right)$ if

$$
t \alpha_{k}^{2}\left[\left(t \alpha_{k-1}^{4}+1\right)+\left(\alpha_{k}^{2}+t \alpha_{k} \alpha_{k-1}^{2}\right)\right]=0,
$$

as seen by eliminating $\alpha_{k+1}$ by means of (119). But the quantity in brackets reduces to $\beta_{k}+\beta_{k} \equiv 0$ by (119) with $k$ replaced by $k-1$, and (120). In view of (118), relation (120) holds for $k=2$ and $k=3$. Hence the induction is complete. Thus

$$
\alpha_{k+1}=1+t \alpha_{k} \alpha_{k-1}^{2}, \quad \alpha_{k+1}=\alpha_{k}^{2}+t \alpha_{k-1}^{4} .
$$

Upon equating the second members, we obtain an equation designated (121').

We next prove the following relation between two $\alpha$ 's :

$$
\alpha_{k+1}+t \alpha_{k+1}^{2} \alpha_{k}^{2}+\alpha_{k}^{2}=t^{2 k-1} \text {. }
$$

It holds for $k=1$ and $k=2$ by (118). Assume it true as far as $k-1$. Then

$$
\alpha_{k}+t \alpha_{k}^{2} \alpha_{k-1}^{2}+\alpha_{k-1}^{2}=t^{2^{k-1-1}} \text {. }
$$

Multiplying the square of the latter by $t$, we see that (122) is true if

$$
\left(\alpha_{k+1}+\alpha_{k}^{2}+t \alpha_{k-1}^{4}\right)+t \alpha_{k}^{2}\left(\alpha_{k+1}^{2}+1+t^{2} \alpha_{k}^{2} \alpha_{k-1}^{4}\right)=0 .
$$

The first part vanishes by $\left(121_{2}\right)$, the second by the square of $\left(121_{1}\right)$.

By (117) for $k=n$, a root of (116) belongs to the $G F\left[2^{n}\right]$ if and only if it satisfies also $\alpha_{n}^{2} z+\beta_{n}=0$; in fact, $z \neq 0$ since $t \neq 0$. If $a_{n}=0$, then $\beta_{n}=0$ by (120) for $k=n$, and the cubic has three distinct roots in the field. Hence $\alpha_{n} \neq 0$ is a necessary condition that the cubic shall have a single root in the field. By (120),

$$
z=1+t \alpha_{n-1}^{2} a_{n}^{-1} .
$$

This uniquely determined value is actually a root of (116) if and only if $R_{n}=0$, where

$$
R_{k}=\alpha_{k}^{3}+t \alpha_{k} \alpha_{k-1}^{4}+t^{2} \alpha_{k-1}^{8} .
$$

Multiply (121') by $\alpha_{k},\left(121_{2}\right)$ by $t \alpha_{k-1}^{2}$, and add. Thus

$$
R_{k}=\alpha_{k}+t \alpha_{k+1} \alpha_{k-1}^{2} \text {. }
$$


In $R_{k+1}$, obtained from $\left(124^{\prime}\right)$, we replace $\alpha_{k+2}$ by its value from $\left(121_{1}\right)$. Then

$$
R_{k}^{2}+R_{k+1}=(1+t) \alpha_{k}^{2}+\alpha_{k+1} S, \quad S=1+t^{2} \alpha_{k}^{4}+t^{2} \alpha_{k+1} \alpha_{k-1}^{4} .
$$

In $S$ we replace $\alpha_{k+1}$ by its value $\left(121_{2}\right)$, and then add the square of $\left(121^{\prime}\right)$ :

$$
S=(1+t)\left\{t \alpha_{k}^{4}+\left(\alpha_{k}^{4}+t^{2} \alpha_{k-1}^{8}\right)\right\} .
$$

The quantity in the last parenthesis equals $\alpha_{k+1}^{2}$ by $\left(121_{2}\right)$. Hence

$$
R_{k}^{2}+R_{k+1}=(1+t)\left(\alpha_{k}^{2}+t \alpha_{k}^{4} \alpha_{k+1}+\alpha_{k+1}^{3}\right) \text {. }
$$

To the last factor add the product of $\alpha_{k+1}$ by $\left(121^{\prime}\right)$ with $k$ replaced by $k+1$; there results the left member of (122). Hence

$$
R_{k}^{2}+R_{k+1}=t^{2^{k}}+t^{2^{k}-1} \text {. }
$$

Raise to the power $2^{i}$ the equation (125) for $k=n-i-1$. Then

$$
R_{n-i}^{2^{i}}+R_{n-i-1}^{2^{+1}}=t^{2^{n-1}}+t^{2^{n-1}-2^{4}} \text {. }
$$

Forming the sum of the latter for $i=0,1, \cdots, n-3$, we get

$$
R_{n}=(n-1) t^{2^{n-1}}+\sum_{i=0}^{n-1} t^{2^{n-1}-2^{i}},
$$

upon replacing $R_{2}$ by its value $t^{2}+t+1$. Hence, finally,

$$
R_{n}=t^{2^{n-1}}\left\{n-1+\chi\left(t^{-1}\right)\right\},
$$

where $\chi$ is the function (42). We note that for $R_{n}=0, t \neq 0$, then $\alpha_{n}$ can not vanish ; for, if $80,(124)$, with $k=n$, would give $\alpha_{n-1}=0$, in contradiction with $\left(121_{1}\right)$ for $k=n-1$. Hence we have the

TheOREM. The cubic $z^{3}=z+t$, with $t \neq 0$, has one and but one root in the $G F\left[2^{n}\right]$ if and only if $\chi\left(t^{-1}\right)=n-1$.

The unique root $z=\rho$ is given by (123), in which the $\alpha$ 's are defined by the recursion formulae (121) with the initial values (118). Removing the factor $z-\rho$ from (116), we obtain $z^{2}+\rho z+\rho^{2}+1$. Since the latter is irreducible, $\chi\left(1+\rho^{-2}\right)=1$, whence $\chi\left(\rho^{-1}\right)=n-1$. When $t$ ranges over the elements $\neq 0$ of the $G F\left[2^{n}\right]$ for which $z^{3}=z+t$ has an unique root $\rho_{t}$, the latter ranges over the same elements; the function $z^{3}-z$ represents a substitution on these elements.

27. The condition on $t$ becomes $\chi\left(1+t^{-2}\right)=1$ upon applying

$$
\chi(1+s)=n+\chi(s), \quad \chi(s)=\chi\left(s^{2}\right) .
$$

We insert the value of $t$ from (115). Hence the condition is

$$
\chi\left(1+g^{-2} e^{3}\right)=1
$$

if $e g \neq 0$. For $e=0, g \neq 0$, this is equivalent to the condition, obtained 
above for this case, that $n$ is odd. Hence for $g \neq 0, e$ arbitrary, the condition is

$$
\chi(\epsilon)=1, \quad \epsilon=\left(g^{2}+e^{3}\right) g^{2^{n}-3},
$$

where (and below) $2^{n}-3$ is to be replaced by unity if $n=1$.

Let $H$ be the characteristic absolute invariant for the class of cubic forms having a linear and an irreducible quadratic factor, so that $H=1$ for such a cubic, while $H=0$ for all others. If $a_{0} \neq 0$, we have $H=\chi(\epsilon), \epsilon$ being defined by (128). For, if $g=0$, then $D=0$, so that $H=0$ by definition. Hence in every case

$$
H=\chi(\epsilon)+m\left(a_{0}^{\mu}-1\right) \quad\left(\mu=2^{n}-1\right) .
$$

Let $a_{0}=0$; then $m$ equals the left member of (113). Without altering $H$, we may add $\chi\left(a_{0} a_{3}^{2} a_{2}^{2^{n}-4}\right)$ to $m$. Inserting the values (114) of $e, g$, we get

$$
H=\chi(\lambda), \quad \lambda=\Delta\left\{\left(a_{1}^{6}+a_{1}^{4} a_{0} a_{2}+a_{0}^{3} a_{2}^{3}+a_{0}^{4} a_{3}^{2}\right) a_{0} a_{0}^{\nu} \Delta^{\nu}+a_{3} a_{2}^{\nu}\left(a_{0}^{\mu}-1\right)\right\},
$$

where $\mu=2^{n}-1, \nu=2^{n}-4$, the latter being replaced by zero if $n=1$ : while

$$
\Delta=a_{0} a_{3}+a_{1} a_{2}=D^{t}=D^{2^{n-1}} .
$$

For $n=1$ and $n=2, \lambda$ becomes

$$
\begin{gathered}
a_{0} a_{3}+a_{0} a_{1} a_{2}+a_{0} a_{1} a_{3}+a_{0} a_{2} a_{3}+a_{1} a_{2} a_{3}+a_{0} a_{1} a_{2} a_{3}, \\
a_{0} a_{1} a_{2}+a_{0} a_{1}^{3} a_{3}^{2}+a_{0} a_{2}^{3} a_{3}^{2}+a_{1} a_{2} a_{3}+a_{0} a_{1}^{2} a_{2}^{2} a_{3},
\end{gathered}
$$

and $H=\Delta(1+R+K), \Delta R$, respectively, where $R$ and $K$ are invariants given in these Transactions, vol. 8 (1907), p. 222, p. 230.

For general $n$, linear combinations of the $D^{i} H(i=0, \ldots, \mu-1)$ give characteristic invariants under $G_{1}$ for the classes defined by $(96)$, whose discriminant $D$ is $m^{4}$.

TheORem. The $d+2+3 \mu$ invariants (111) and $D^{i} H(i=0, \ldots, \mu-1)$ give a complete set of linearly independent invariants of the binary cubic form in the $G F\left[2^{n}\right]$.

The product of any two of these invariants can be reduced to a linear combination of them by means of relations (112) and

$$
H^{2}=H, \quad D^{\mu} H=H, \quad H I_{0}=H Q=H J=H \pi=H E=0 .
$$

The University of Chicago, December 1, 1908. 